\title{
Supporting Data for
}

\section{Substituent Effects on Nitrosyl Iron Corrole Complexes $\mathrm{Fe}\left(\mathrm{Ar}_{3} \mathrm{C}\right)(\mathrm{NO})$.}

\author{
Crisjoe A. Joseph, Matthew S. Lee, Alexei V. Iretski, Guang Wu and Peter C. Ford* \\ Contribution from the Department of Chemistry and Biochemistry, University of California, \\ Santa Barbara, Santa Barbara, California 93106-9510, USA \\ e.mail:ford@chem.ucsb.edu
}

Contribution from the Department of Chemistry and Biochemistry, University of California, Santa Barbara, Santa Barbara, California 93106-9510, USA

\section{Contents:}

\section{List of Supplemental Figures}

Figure S1. Crystal packing structure of $\mathrm{Fe}(\mathrm{TTC})(\mathrm{NO})$. Green = iron; red = oxygen; blue = nitrogen; grey $=$ corrole carbon, purple $=$ solvent (benzene) carbon and yellow $=$ unit cell.

Figure S2. Crystal packing structure of $\mathrm{Fe}(\mathrm{TMOPC})(\mathrm{NO})$. Green $=$ iron; red = oxygen; blue = nitrogen; grey $=$ corrole carbon, purple $=$ solvent $($ benzene) carbon and yellow $=$ unit cell

\section{List of Supplemental Tables}

Tables S1a--S1f: X-ray crystallography data for the structure of Fe(TTC)(NO) (TTC = the 5,10,15-tris(4-tolyl)corrolato trianion)

Tables S2a - S2f: X-ray crystallography data for the structure of Fe(TMOPC)(NO) (TMOPC = the 5,10,15-tris(4-methoxyphenyl)corrolato trianion)

Table S-3: Computational results for $[\mathrm{Fe}(\mathrm{C})(\mathrm{NO})]$. 
Figure S1. Crystal packing structure of Fe(TTC)(NO). Green = iron; red = oxygen; blue = nitrogen; grey $=$ corrole carbon, purple $=$ solvent $($ benzene $)$ carbon and yellow $=$ unit cell.

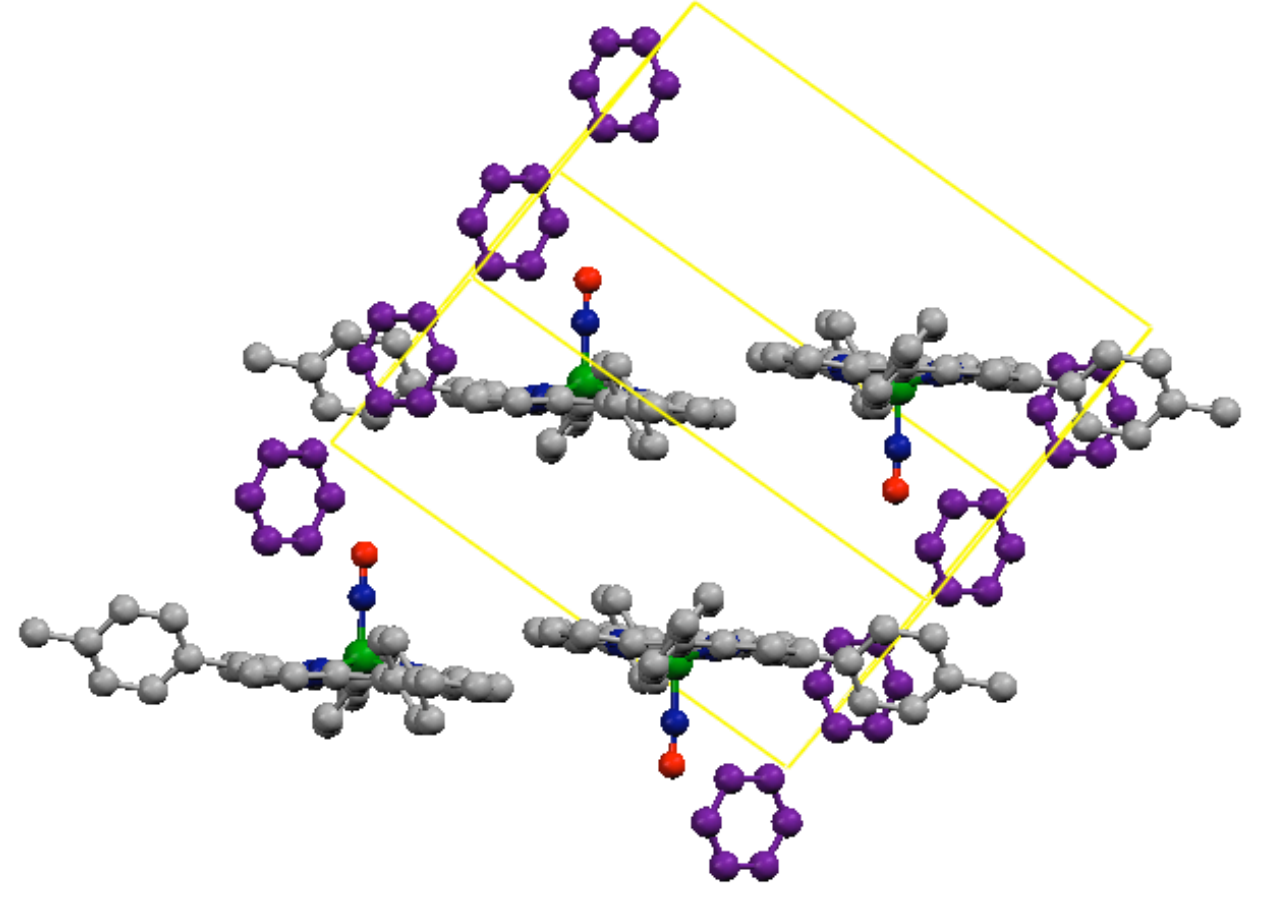


Figure S2. Crystal packing structure of Fe(TMOPC)(NO). Green = iron; red = oxygen; blue = nitrogen; grey $=$ corrole carbon, purple $=$ solvent (benzene) carbon and yellow $=$ unit cell.

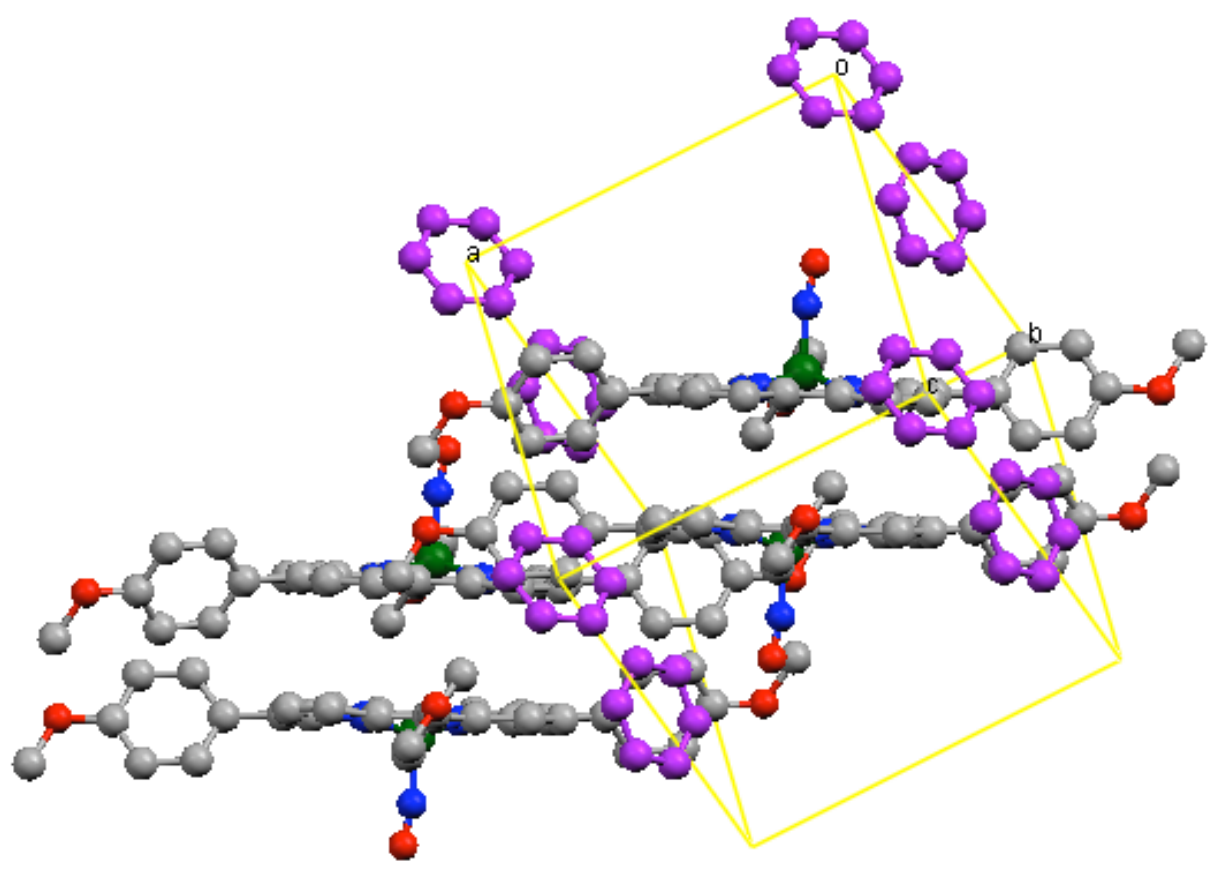


Table S1a. Crystal Data and Structure Refinement for Fe(TTC)(NO) (TTC = the 5,10,15-tris(4tolyl)corrolato trianion).

\begin{tabular}{|c|c|}
\hline \multirow[b]{2}{*}{ Empirical formula } & Fe(TTC)NO \\
\hline & $\mathrm{C}_{46} \mathrm{H}_{35} \mathrm{FeN}_{5} \mathrm{O}$ \\
\hline Formula weight & 729.64 \\
\hline Temperature & $118(1) \mathrm{K}$ \\
\hline Wavelength & $0.71073 \AA$ \\
\hline Crystal system, space group & Triclinic, $\mathrm{P}-1$ \\
\hline Unit cell dimensions & $a=10.652(1) \AA$ \\
\hline & $b=10.923(1) \AA$ \\
\hline & $c=15.285(2) \AA$ \\
\hline & $\alpha=87.514(2) \mathrm{deg}$. \\
\hline & $\beta=85.987(2) \mathrm{deg}$. \\
\hline & $\gamma=84.216(2) \mathrm{deg}$ \\
\hline Volume & $1763.9(4) \AA^{3}$ \\
\hline Z, Calculated density & $2,1.374 \mathrm{Mg} / \mathrm{m}^{3}$ \\
\hline Absorption coefficient & $0.473 \mathrm{~mm}^{-1}$ \\
\hline $\mathrm{F}(000)$ & 760 \\
\hline Crystal size & $0.3 \times 0.3 \times 0.1 \mathrm{~m}$ \\
\hline Theta range for data collection & 1.34 to $28.15 \mathrm{deg}$ \\
\hline Limiting indices & $-14<=\mathrm{h}<=13,-14<=\mathrm{k}<=13,-19<=\mathrm{k}<=19$ \\
\hline Reflections collected / unique & $15172 / 7541[\mathrm{R}(\mathrm{i} \mathrm{nt})=0.0264]$ \\
\hline Completeness to theta $=28.15$ & $87.10 \%$ \\
\hline Absorption correction & None \\
\hline Refinement method & Full-matrix least -squares on $\mathrm{F}^{2}$ \\
\hline Data / restraints / parameters & $7541 / 0 / 618$ \\
\hline Goodness-of-fit on $\mathrm{F}^{2}$ & 1.036 \\
\hline Final $\mathrm{R}$ indices $[\mathrm{I}>2 \operatorname{sigma}(\mathrm{I})]$ & $\mathrm{R} 1=0.0431, \mathrm{wR} 2=0.1105$ \\
\hline $\mathrm{R}$ indices (all data) & $\mathrm{R} 1=0.0612, \mathrm{wR} 2=0.1190$ \\
\hline Largest diff. peak and hole & 0.666 and $-0.589 \AA^{3}$ \\
\hline
\end{tabular}


Table S1b. Atomic Coordinates and Equivalent Isotropic Displacement Parameters for $\mathrm{Fe}(\mathrm{TTC})(\mathrm{NO})(\mathrm{TTC}=$ the 5,10,15-tris(4-tolyl)corrolato trianion).

\begin{tabular}{|c|c|c|c|c|}
\hline & $x\left(x 10^{4}\right)$ & $y\left(x 10^{4}\right)$ & $\mathrm{z}\left(\mathrm{x} \quad 10^{4}\right)$ & $U_{\text {eq }}\left(\AA^{2} \times 10^{3}\right)^{a}$ \\
\hline $\mathrm{C}(1)$ & $9546(2)$ & $3741(2)$ & $5603(1)$ & $20(1)$ \\
\hline $\mathrm{C}(2)$ & $10409(2)$ & 2901(2) & $5118(2)$ & $22(1)$ \\
\hline $\mathrm{C}(3)$ & $11472(2)$ & $2699(2)$ & $5576(2)$ & $22(1)$ \\
\hline$C(4)$ & $11282(2)$ & $3425(2)$ & $6346(1)$ & $20(1)$ \\
\hline$C(5)$ & $12107(2)$ & $3671(2)$ & $6967(1)$ & $20(1)$ \\
\hline$C(6)$ & $11760(2)$ & $4565(2)$ & $7610(1)$ & $19(1)$ \\
\hline$C(7)$ & $12502(2)$ & $4813(2)$ & $8318(1)$ & 21(1) \\
\hline $\mathrm{C}(8)$ & $11816(2)$ & $5673(2)$ & $8819(2)$ & $24(1)$ \\
\hline $\mathrm{C}(9)$ & $10640(2)$ & $5988(2)$ & $8426(1)$ & $20(1)$ \\
\hline$C(10)$ & $9648(2)$ & $6846(2)$ & $8720(1)$ & $20(1)$ \\
\hline $\mathrm{C}(11)$ & $8523(2)$ & $7142(2)$ & $8292(1)$ & $21(1)$ \\
\hline $\mathrm{C}(12)$ & $7542(2)$ & $8101(2)$ & $8500(2)$ & $22(1)$ \\
\hline$C(13)$ & $6638(2)$ & $8063(2)$ & $7913(2)$ & $22(1)$ \\
\hline$C(14)$ & $7041(2)$ & $7082(2)$ & $7341(1)$ & $20(1)$ \\
\hline$C(15)$ & $6379(2)$ & $6747(2)$ & $6624(1)$ & $20(1)$ \\
\hline$C(16)$ & $6892(2)$ & $5843(2)$ & 6061(1) & $18(1)$ \\
\hline$C(17)$ & $6457(2)$ & $5416(2)$ & $5267(1)$ & $21(1)$ \\
\hline C(18) & $7350(2)$ & $4538(2)$ & $4942(2)$ & $22(1)$ \\
\hline$C(19)$ & $8346(2)$ & $4407(2)$ & $5527(1)$ & $20(1)$ \\
\hline $\mathrm{C}(20)$ & $13400(2)$ & $3008(2)$ & $6933(1)$ & $19(1)$ \\
\hline $\mathrm{C}(21)$ & $13584(2)$ & $1731(2)$ & $6896(1)$ & $22(1)$ \\
\hline$C(22)$ & $14796(2)$ & $1118(2)$ & $6844(2)$ & $24(1)$ \\
\hline$C(23)$ & $15858(2)$ & $1756(2)$ & $6822(1)$ & $24(1)$ \\
\hline$C(24)$ & $15673(2)$ & $3036(2)$ & $6844(2)$ & $25(1)$ \\
\hline$C(25)$ & $14469(2)$ & $3655(2)$ & $6900(2)$ & $23(1)$ \\
\hline$C(26)$ & $17175(3)$ & $1102(3)$ & $6792(2)$ & $35(1)$ \\
\hline$C(27)$ & $9737(2)$ & $7477(2)$ & $9554(1)$ & $20(1)$ \\
\hline$C(28)$ & $10705(2)$ & $8204(2)$ & $9685(2)$ & $22(1)$ \\
\hline$C(29)$ & $10714(2)$ & $8813(2)$ & $10459(2)$ & $24(1)$ \\
\hline$C(30)$ & $9777(2)$ & $8712(2)$ & 11129(1) & $24(1)$ \\
\hline$C(31)$ & $8826(2)$ & $7977(2)$ & $11003(2)$ & $24(1)$ \\
\hline$C(32)$ & $8802(2)$ & $7366(2)$ & $10229(2)$ & $25(1)$ \\
\hline$C(33)$ & $9779(3)$ & $9381(3)$ & $11968(2)$ & $32(1)$ \\
\hline$C(34)$ & $5107(2)$ & $7396(2)$ & $6478(1)$ & $21(1)$ \\
\hline$C(35)$ & $4104(2)$ & $7333(2)$ & $7107(2)$ & $24(1)$ \\
\hline$C(36)$ & $2904(2)$ & $7904(2)$ & $6954(2)$ & $25(1)$ \\
\hline$C(37)$ & $2682(2)$ & $8575(2)$ & $6176(2)$ & $24(1)$ \\
\hline $\mathrm{C}(38)$ & $3688(2)$ & $8645(2)$ & $5553(2)$ & $26(1)$ \\
\hline$C(39)$ & $4881(2)$ & $8063(2)$ & $5696(2)$ & $24(1)$ \\
\hline$C(40)$ & $1389(2)$ & $9200(3)$ & $6007(2)$ & $30(1)$ \\
\hline $\mathrm{C}(41)$ & $5867(3)$ & $807(3)$ & $9804(3)$ & $66(1)$ \\
\hline$C(42)$ & $4928(4)$ & $712(4)$ & $9251(2)$ & $60(1)$ \\
\hline
\end{tabular}




$\begin{array}{lccrr}\mathrm{C}(43) & 4071(4) & -86(3) & 9453(3) & 69(1) \\ \mathrm{C}(44) & 5439(3) & 4072(3) & 9439(2) & 39(1) \\ \mathrm{C}(45) & 5767(3) & 5249(3) & 9264(2) & 38(1) \\ \mathrm{C}(46) & 5327(3) & 6179(3) & 9819(2) & 39(1) \\ \mathrm{Fe} & 9079(1) & 5017(1) & 7164(1) & 18(1) \\ \mathrm{N}(1) & 8476(2) & 3987(2) & 7843(1) & 21(1) \\ \mathrm{N}(2) & 10089(2) & 4036(2) & 6337(1) & 20(1) \\ \mathrm{N}(3) & 10623(2) & 5302(2) & 7683(1) & 19(1) \\ \mathrm{N}(4) & 8211(2) & 6540(2) & 7566(1) & 20(1) \\ \mathrm{N}(5) & 8051(2) & 5194(2) & 6192(1) & 19(1) \\ \mathrm{O} & 8038(2) & 3233(2) & 8293(1) & 41(1) \\ {[a] \mathrm{U}_{\text {eq }} \text { is defined as one third of the trace of the orthogonalized } \mathrm{U}_{\mathrm{ij}} \text { tensor. }}\end{array}$


Table S1c. Bond Lengths for Fe(TTC)(NO) (TTC = the 5,10,15-tris(4-tolyl)corrolato trianion). ${ }^{a}$

\begin{tabular}{ll}
\hline Bond & Length $\AA$ \\
\hline $\mathrm{C}(1)-\mathrm{N}(2)$ & $1.362(3)$ \\
$\mathrm{C}(1)-\mathrm{C}(19)$ & $1.415(3)$ \\
$\mathrm{C}(1)-\mathrm{C}(2)$ & $1.425(3)$ \\
$\mathrm{C}(2)-\mathrm{C}(3)$ & $1.366(3)$ \\
$\mathrm{C}(2)-\mathrm{H}(2)$ & $0.97(3)$ \\
$\mathrm{C}(3)-\mathrm{C}(4)$ & $1.439(3)$ \\
$\mathrm{C}(3)-\mathrm{H}(3)$ & $0.93(3)$ \\
$\mathrm{C}(4)-\mathrm{N}(2)$ & $1.375(3)$ \\
$\mathrm{C}(4)-\mathrm{C}(5)$ & $1.390(3)$ \\
$\mathrm{C}(5)-\mathrm{C}(6)$ & $1.420(3)$ \\
$\mathrm{C}(5)-\mathrm{C}(20)$ & $1.489(3)$ \\
$\mathrm{C}(6)-\mathrm{N}(3)$ & $1.385(3)$ \\
$\mathrm{C}(6)-\mathrm{C}(7)$ & $1.434(3)$ \\
$\mathrm{C}(7)-\mathrm{C}(8)$ & $1.360(3)$ \\
$\mathrm{C}(7)-\mathrm{H}(7)$ & $0.96(3)$ \\
$\mathrm{C}(8)-\mathrm{C}(9)$ & $1.432(3)$ \\
$\mathrm{C}(8)-\mathrm{H}(8)$ & $0.96(3)$ \\
$\mathrm{C}(9)-\mathrm{N}(3)$ & $1.389(3)$ \\
$\mathrm{C}(9)-\mathrm{C}(10)$ & $1.403(3)$ \\
$\mathrm{C}(10)-\mathrm{C}(11)$ & $1.408(3)$ \\
$\mathrm{C}(10)-\mathrm{C}(27)$ & $1.489(3)$ \\
$\mathrm{C}(11)-\mathrm{N}(4)$ & $1.391(3)$ \\
$\mathrm{C}(11)-\mathrm{C}(12)$ & $1.432(3)$ \\
$\mathrm{C}(12)-\mathrm{C}(13)$ & $1.367(3)$ \\
$\mathrm{C}(12)-\mathrm{H}(12)$ & $0.99(2)$ \\
$\mathrm{C}(13)-\mathrm{C}(14)$ & $1.425(3)$ \\
$\mathrm{C}(13)-\mathrm{H}(13)$ & $0.96(2)$ \\
$\mathrm{C}(14)-\mathrm{N}(4)$ & $1.383(3)$ \\
$\mathrm{C}(14)-\mathrm{C}(15)$ & $1.425(3)$ \\
$\mathrm{C}(15)-\mathrm{C}(16)$ & $1.383(3)$ \\
$\mathrm{C}(15)-\mathrm{C}(34)$ & $1.491(3)$ \\
$\mathrm{C}(16)-\mathrm{N}(5)$ & $1.383(3)$ \\
$\mathrm{C}(16)-\mathrm{C}(17)$ & $1.441(3)$ \\
$\mathrm{C}(17)-\mathrm{C}(18)$ & $1.365(3)$ \\
$\mathrm{C}(17)-\mathrm{H}(17)$ & $0.95(3)$ \\
$\mathrm{C}(18)-\mathrm{C}(19)$ & $1.427(3)$ \\
$\mathrm{C}(18)-\mathrm{H}(18)$ & $0.95(3)$ \\
$\mathrm{C}(19)-\mathrm{N}(5)$ & $1.359(3)$ \\
$\mathrm{C}(20)-\mathrm{C}(21)$ & $1.392(3)$ \\
$\mathrm{C}(20)-\mathrm{C}(25)$ & $1.397(3)$ \\
$\mathrm{C}(21)-\mathrm{C}(22)$ & $1.392(3)$ \\
$\mathrm{C}(21)-\mathrm{H}(21)$ & $0.97(2)$ \\
$\mathrm{C}(22)-\mathrm{C}(23)$ & $1.384(3)$ \\
&
\end{tabular}




$\begin{array}{ll}\mathrm{C}(22)-\mathrm{H}(22) & 0.91(3) \\ \mathrm{C}(23)-\mathrm{C}(24) & 1.393(3) \\ \mathrm{C}(23)-\mathrm{C}(26) & 1.507(3) \\ \mathrm{C}(24)-\mathrm{C}(25) & 1.388(3) \\ \mathrm{C}(24)-\mathrm{H}(24) & 0.95(3) \\ \mathrm{C}(25)-\mathrm{H}(25) & 0.99(2) \\ \mathrm{C}(26)-\mathrm{H}(26 \mathrm{~A}) & 0.96(3) \\ \mathrm{C}(26)-\mathrm{H}(26 \mathrm{~B}) & 0.96(3) \\ \mathrm{C}(26)-\mathrm{H}(26 \mathrm{C}) & 0.99(4) \\ \mathrm{C}(27)-\mathrm{C}(28) & 1.393(3) \\ \mathrm{C}(27)-\mathrm{C}(32) & 1.394(3) \\ \mathrm{C}(28)-\mathrm{C}(29) & 1.382(3) \\ \mathrm{C}(28)-\mathrm{H}(28) & 0.92(3) \\ \mathrm{C}(29)-\mathrm{C}(30) & 1.388(3) \\ \mathrm{C}(29)-\mathrm{H}(29) & 0.94(3) \\ \mathrm{C}(30)-\mathrm{C}(31) & 1.383(3) \\ \mathrm{C}(30)-\mathrm{C}(33) & 1.504(3) \\ \mathrm{C}(31)-\mathrm{C}(32) & 1.387(3) \\ \mathrm{C}(31)-\mathrm{H}(31) & 0.92(3) \\ \mathrm{C}(32)-\mathrm{H}(32) & 0.91(3) \\ \mathrm{C}(33)-\mathrm{H}(33 \mathrm{~A}) & 0.94(3) \\ \mathrm{C}(33)-\mathrm{H}(33 \mathrm{~B}) & 0.96(3) \\ \mathrm{C}(33)-\mathrm{H}(33 \mathrm{C}) & 0.94(3) \\ \mathrm{C}(34)-\mathrm{C}(35) & 1.392(3) \\ \mathrm{C}(34)-\mathrm{C}(39) & 1.395(3) \\ \mathrm{C}(35)-\mathrm{C}(36) & 1.395(3) \\ \mathrm{C}(35)-\mathrm{H}(35) & 1.00(3) \\ \mathrm{C}(36)-\mathrm{C}(37) & 1.391(3) \\ \mathrm{C}(36)-\mathrm{H}(36) & 0.93(3) \\ \mathrm{C}(37)-\mathrm{C}(38) & 1.388(3) \\ \mathrm{C}(37)-\mathrm{C}(40) & 1.508(3) \\ \mathrm{C}(38)-\mathrm{C}(39) & 1.390(3) \\ \mathrm{C}(38)-\mathrm{H}(38) & 0.96(3) \\ \mathrm{C}(39)-\mathrm{H}(39) & 0.96(3) \\ \mathrm{C}(40)-\mathrm{H}(40 \mathrm{~A}) & 0.97(3) \\ \mathrm{C}(40)-\mathrm{H}(40 \mathrm{~B}) & 0.96(3) \\ \mathrm{C}(40)-\mathrm{H}(40 \mathrm{C}) & 0.96(3) \\ \mathrm{C}(41)-\mathrm{C}(43) \# 1 & 1.354(6) \\ \mathrm{C}(41)-\mathrm{C}(42) & 1.370(6) \\ \mathrm{C}(41)-\mathrm{H}(41) & 0.94(4) \\ \mathrm{C}(42)-\mathrm{C}(43) & 1.336(6) \\ \mathrm{C}(42)-\mathrm{H}(42) & 0.92(4) \\ \mathrm{C}(43) & 1.354(6) \\ & 0.92(4) \\ \mathrm{C}(43) & 1.384(4) \\ \end{array}$




$\begin{array}{ll}\mathrm{C}(44)-\mathrm{H}(44) & 0.95(3) \\ \mathrm{C}(45)-\mathrm{C}(46) & 1.377(4) \\ \mathrm{C}(45)-\mathrm{H}(45) & 0.98(3) \\ \mathrm{C}(46)-\mathrm{C}(44) \# 2 & 1.384(4) \\ \mathrm{C}(46)-\mathrm{H}(46) & 0.93(3) \\ \mathrm{Fe}-\mathrm{N}(1) & 1.645(2) \\ \mathrm{Fe}-\mathrm{N}(2) & 1.897(2) \\ \mathrm{Fe}-\mathrm{N}(5) & 1.899(2) \\ \mathrm{Fe}-\mathrm{N}(4) & 1.923(2) \\ \mathrm{Fe}-\mathrm{N}(3) & 1.934(2) \\ \mathrm{N}(1)-\mathrm{O} & 1.162(2)\end{array}$

[a] Symmetry transformations used to generate equivalent atoms: \#1 -x+1,-y,-z+2 \#2-x+1,-y+1,-z+2 


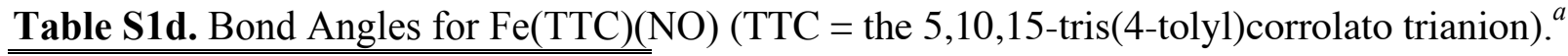

\begin{tabular}{ll}
\hline Bond & $\begin{array}{l}\text { Length } \\
\text { (deg) }\end{array}$ \\
\hline N(2)-C(1)-C(19) & $111.5(2)$ \\
N(2)-C(1)-C(2) & $109.0(2)$ \\
C(19)-C(1)-C(2) & $139.4(2)$ \\
C(3)-C(2)-C(1) & $106.9(2)$ \\
C(3)-C(2)-H(2) & $126(2)$ \\
C(1)-C(2)-H(2) & $127(2)$ \\
C(2)-C(3)-C(4) & $108.1(2)$ \\
C(2)-C(3)-H(3) & $127(2)$ \\
C(4)-C(3)-H(3) & $125(2)$ \\
N(2)-C(4)-C(5) & $121.0(2)$ \\
N(2)-C(4)-C(3) & $107.9(2)$ \\
C(5)-C(4)-C(3) & $131.4(2)$ \\
C(4)-C(5)-C(6) & $121.6(2)$ \\
C(4)-C(5)-C(20) & $118.9(2)$ \\
C(6)-C(5)-C(20) & $119.5(2)$ \\
N(3)-C(6)-C(5) & $125.7(2)$ \\
N(3)-C(6)-C(7) & $108.6(2)$ \\
C(5)-C(6)-C(7) & $125.7(2)$ \\
C(8)-C(7)-C(6) & $108.0(2)$ \\
C(8)-C(7)-H(7) & $127(2)$ \\
C(6)-C(7)-H(7) & $125(2)$ \\
C(7)-C(8)-C(9) & $107.5(2)$ \\
C(7)-C(8)-H(8) & $127(2)$ \\
C(9)-C(8)-H(8) & $125(2)$ \\
N(3)-C(9)-C(10) & $124.1(2)$ \\
N(3)-C(9)-C(8) & $108.9(2)$ \\
C(10)-C(9)-C(8) & $127.0(2)$ \\
C(9)-C(10)-C(11) & $124.5(2)$ \\
C(9)-C(10)-C(27) & $119.5(2)$ \\
C(11)-C(10)-C(27) & $116.0(2)$ \\
N(4)-C(11)-C(10) & $123.9(2)$ \\
N(4)-C(11)-C(12) & $109.0(2)$ \\
C(10)-C(11)-C(12) & $127.2(2)$ \\
C(13)-C(12)-C(11) & $107.3(2)$ \\
C(13)-C(12)-H(12) & $128(1)$ \\
C(11)-C(12)-H(12) & $124(1)$ \\
C(12)-C(13)-C(14) & $107.7(2)$ \\
C(12)-C(13)-H(13) & $126(1)$ \\
C(14)-C(13)-H(13) & $126(1)$ \\
N(4)-C(14)-C(15) & $125.0(2)$ \\
N(4)-C(14)-C(13) & $109.3(2)$ \\
C(15)-C(14)-C(13) & $125.6(2)$ \\
C(16)-C(15)-C(14) & $121.6(2)$ \\
&
\end{tabular}




$\begin{array}{ll}\mathrm{C}(16)-\mathrm{C}(15)-\mathrm{C}(34) & 119.5(2) \\ \mathrm{C}(14)-\mathrm{C}(15)-\mathrm{C}(34) & 118.9(2) \\ \mathrm{N}(5)-\mathrm{C}(16)-\mathrm{C}(15) & 121.2(2) \\ \mathrm{N}(5)-\mathrm{C}(16)-\mathrm{C}(17) & 107.2(2) \\ \mathrm{C}(15)-\mathrm{C}(16)-\mathrm{C}(17) & 131.6(2) \\ \mathrm{C}(18)-\mathrm{C}(17)-\mathrm{C}(16) & 107.9(2) \\ \mathrm{C}(18)-\mathrm{C}(17)-\mathrm{H}(17) & 128(2) \\ \mathrm{C}(16)-\mathrm{C}(17)-\mathrm{H}(17) & 124(2) \\ \mathrm{C}(17)-\mathrm{C}(18)-\mathrm{C}(19) & 107.2(2) \\ \mathrm{C}(17)-\mathrm{C}(18)-\mathrm{H}(18) & 128(2) \\ \mathrm{C}(19)-\mathrm{C}(18)-\mathrm{H}(18) & 125(2) \\ \mathrm{N}(5)-\mathrm{C}(19)-\mathrm{C}(1) & 111.8(2) \\ \mathrm{N}(5)-\mathrm{C}(19)-\mathrm{C}(18) & 109.0(2) \\ \mathrm{C}(1)-\mathrm{C}(19)-\mathrm{C}(18) & 139.2(2) \\ \mathrm{C}(21)-\mathrm{C}(20)-\mathrm{C}(25) & 117.9(2) \\ \mathrm{C}(21)-\mathrm{C}(20)-\mathrm{C}(5) & 121.2(2) \\ \mathrm{C}(25)-\mathrm{C}(20)-\mathrm{C}(5) & 120.8(2) \\ \mathrm{C}(20)-\mathrm{C}(21)-\mathrm{C}(22) & 121.0(2) \\ \mathrm{C}(20)-\mathrm{C}(21)-\mathrm{H}(21) & 119(1) \\ \mathrm{C}(22)-\mathrm{C}(21)-\mathrm{H}(21) & 120(1) \\ \mathrm{C}(23)-\mathrm{C}(22)-\mathrm{C}(21) & 121.3(2) \\ \mathrm{C}(23)-\mathrm{C}(22)-\mathrm{H}(22) & 122(2) \\ \mathrm{C}(21)-\mathrm{C}(22)-\mathrm{H}(22) & 117(2) \\ \mathrm{C}(22)-\mathrm{C}(23)-\mathrm{C}(24) & 117.7(2) \\ \mathrm{C}(22)-\mathrm{C}(23)-\mathrm{C}(26) & 121.8(2) \\ \mathrm{C}(24)-\mathrm{C}(23)-\mathrm{C}(26) & 120.4(2) \\ \mathrm{C}(25)-\mathrm{C}(24)-\mathrm{C}(23) & 121.5(2) \\ \mathrm{C}(25)-\mathrm{C}(24)-\mathrm{H}(24) & 118(2) \\ \mathrm{C}(23)-\mathrm{C}(24)-\mathrm{H}(24) & 121(2) \\ \mathrm{C}(24)-\mathrm{C}(25)-\mathrm{C}(20) & 120.6(2) \\ \mathrm{C}(24)-\mathrm{C}(25)-\mathrm{H}(25) & 118(2) \\ \mathrm{C}(20)-\mathrm{C}(25)-\mathrm{H}(25) & 122(2) \\ \mathrm{C}(23)-\mathrm{C}(26)-\mathrm{H}(26 \mathrm{~A}) & 114(2) \\ \mathrm{C}(23)-\mathrm{C}(26)-\mathrm{H}(26 \mathrm{~B}) & 112(2) \\ \mathrm{H}(26 \mathrm{~A})-\mathrm{C}(26)-\mathrm{H}(26 \mathrm{~B}) & 110(3) \\ \mathrm{C}(23)-\mathrm{C}(26)-\mathrm{H}(26 \mathrm{C}) & 108(2) \\ \mathrm{H}(26 \mathrm{~A})-\mathrm{C}(26)-\mathrm{H}(26 \mathrm{C}) & 103(3) \\ \mathrm{H}(26 \mathrm{~B})-\mathrm{C}(26)-\mathrm{H}(26 \mathrm{C}) & 109(3) \\ \mathrm{C}(28)-\mathrm{C}(27)-\mathrm{C}(32) & 118.1(2) \\ \mathrm{C}(28)-\mathrm{C}(27)-\mathrm{C}(10) & 122.8(2) \\ \mathrm{C}(32)-\mathrm{C}(27)-\mathrm{C}(10) & 119.1(2) \\ \mathrm{C}(29)-\mathrm{C}(28)-\mathrm{C}(27) & 120.5(2) \\ \mathrm{C}(29)-\mathrm{C}(28)-\mathrm{H}(28) & 121(2) \\ & 118(2) \\ \mathrm{C}(29)-\mathrm{H}(28) & 117(2) \\ & \end{array}$




$\begin{array}{ll}\mathrm{C}(30)-\mathrm{C}(29)-\mathrm{H}(29) & 121(2) \\ \mathrm{C}(31)-\mathrm{C}(30)-\mathrm{C}(29) & 118.0(2) \\ \mathrm{C}(31)-\mathrm{C}(30)-\mathrm{C}(33) & 120.4(2) \\ \mathrm{C}(29)-\mathrm{C}(30)-\mathrm{C}(33) & 121.6(2) \\ \mathrm{C}(30)-\mathrm{C}(31)-\mathrm{C}(32) & 121.1(2) \\ \mathrm{C}(30)-\mathrm{C}(31)-\mathrm{H}(31) & 122(2) \\ \mathrm{C}(32)-\mathrm{C}(31)-\mathrm{H}(31) & 117(2) \\ \mathrm{C}(31)-\mathrm{C}(32)-\mathrm{C}(27) & 120.8(2) \\ \mathrm{C}(31)-\mathrm{C}(32)-\mathrm{H}(32) & 117(2) \\ \mathrm{C}(27)-\mathrm{C}(32)-\mathrm{H}(32) & 123(2) \\ \mathrm{C}(30)-\mathrm{C}(33)-\mathrm{H}(33 \mathrm{~A}) & 110(2) \\ \mathrm{C}(30)-\mathrm{C}(33)-\mathrm{H}(33 \mathrm{~B}) & 112(2) \\ \mathrm{H}(33 \mathrm{~A})-\mathrm{C}(33)-\mathrm{H}(33 \mathrm{~B}) & 109(3) \\ \mathrm{C}(30)-\mathrm{C}(33)-\mathrm{H}(33 \mathrm{C}) & 111(2) \\ \mathrm{H}(33 \mathrm{~A})-\mathrm{C}(33)-\mathrm{H}(33 \mathrm{C}) & 108(3) \\ \mathrm{H}(33 \mathrm{~B})-\mathrm{C}(33)-\mathrm{H}(33 \mathrm{C}) & 106(3) \\ \mathrm{C}(35)-\mathrm{C}(34)-\mathrm{C}(39) & 118.0(2) \\ \mathrm{C}(35)-\mathrm{C}(34)-\mathrm{C}(15) & 121.1(2) \\ \mathrm{C}(39)-\mathrm{C}(34)-\mathrm{C}(15) & 120.8(2) \\ \mathrm{C}(34)-\mathrm{C}(35)-\mathrm{C}(36) & 120.9(2) \\ \mathrm{C}(34)-\mathrm{C}(35)-\mathrm{H}(35) & 120(2) \\ \mathrm{C}(36)-\mathrm{C}(35)-\mathrm{H}(35) & 119(2) \\ \mathrm{C}(37)-\mathrm{C}(36)-\mathrm{C}(35) & 121.0(2) \\ \mathrm{C}(37)-\mathrm{C}(36)-\mathrm{H}(36) & 120(2) \\ \mathrm{C}(35)-\mathrm{C}(36)-\mathrm{H}(36) & 120(2) \\ \mathrm{C}(38)-\mathrm{C}(37)-\mathrm{C}(36) & 117.9(2) \\ \mathrm{C}(38)-\mathrm{C}(37)-\mathrm{C}(40) & 120.8(2) \\ \mathrm{C}(36)-\mathrm{C}(37)-\mathrm{C}(40) & 121.3(2) \\ \mathrm{C}(37)-\mathrm{C}(38)-\mathrm{C}(39) & 121.4(2) \\ \mathrm{C}(37)-\mathrm{C}(38)-\mathrm{H}(38) & 120(2) \\ \mathrm{C}(39)-\mathrm{C}(38)-\mathrm{H}(38) & 119(2) \\ \mathrm{C}(38)-\mathrm{C}(39)-\mathrm{C}(34) & 120.8(2) \\ \mathrm{C}(38)-\mathrm{C}(39)-\mathrm{H}(39) & 118(2) \\ \mathrm{C}(34)-\mathrm{C}(39)-\mathrm{H}(39) & 121(2) \\ \mathrm{C}(37)-\mathrm{C}(40)-\mathrm{H}(40 \mathrm{~A}) & 111(2) \\ \mathrm{C}(37)-\mathrm{C}(40)-\mathrm{H}(40 \mathrm{~B}) & 109(2) \\ \mathrm{H}(40 \mathrm{~A})-\mathrm{C}(40)-\mathrm{H}(40 \mathrm{~B}) & 109(3) \\ \mathrm{C}(37)-\mathrm{C}(40)-\mathrm{H}(40 \mathrm{C}) & 112(2) \\ \mathrm{H}(40 \mathrm{~A})-\mathrm{C}(40)-\mathrm{H}(40 \mathrm{C}) & 106(2) \\ \mathrm{H}(40 \mathrm{~B})-\mathrm{C}(40)-\mathrm{H}(40 \mathrm{C}) & 109(2) \\ \mathrm{C}(43) \#-\mathrm{C}(41)-\mathrm{C}(42) & 119.9(4) \\ \mathrm{C}(43) \#-\mathrm{C}(\mathrm{C}(41)-\mathrm{H}(41) & 119(2) \\ \mathrm{C}(42)-\mathrm{C}(41)-\mathrm{H}(41) & 121(2) \\ \mathrm{C}(43)-\mathrm{C}(42)-\mathrm{C}(41) & 119.5(3) \\ & 125(3) \\ \mathrm{C}(42) & 116(3) \\ & \end{array}$




$\begin{array}{ll}\mathrm{C}(42)-\mathrm{C}(43)-\mathrm{C}(41) \# 1 & 120.6(3) \\ \mathrm{C}(42)-\mathrm{C}(43)-\mathrm{H}(43) & 114(3) \\ \mathrm{C}(41) \# 1-\mathrm{C}(43)-\mathrm{H}(43) & 125(3) \\ \mathrm{C}(45)-\mathrm{C}(44)-\mathrm{C}(46) \# 2 & 119.8(3) \\ \mathrm{C}(45)-\mathrm{C}(44)-\mathrm{H}(44) & 122(2) \\ \mathrm{C}(46) \# 2-\mathrm{C}(44)-\mathrm{H}(44) & 118(2) \\ \mathrm{C}(44)-\mathrm{C}(45)-\mathrm{C}(46) & 120.3(3) \\ \mathrm{C}(44)-\mathrm{C}(45)-\mathrm{H}(45) & 122(2) \\ \mathrm{C}(46)-\mathrm{C}(45)-\mathrm{H}(45) & 118(2) \\ \mathrm{C}(45)-\mathrm{C}(46)-\mathrm{C}(44) \# 2 & 119.9(3) \\ \mathrm{C}(45)-\mathrm{C}(46)-\mathrm{H}(46) & 119(2) \\ \mathrm{C}(44) \# 2-\mathrm{C}(46)-\mathrm{H}(46) & 121(2) \\ \mathrm{N}(1)-\mathrm{Fe}-\mathrm{N}(2) & 103.05(9) \\ \mathrm{N}(1)-\mathrm{Fe}-\mathrm{N}(5) & 106.30(8) \\ \mathrm{N}(2)-\mathrm{Fe}-\mathrm{N}(5) & 79.19(8) \\ \mathrm{N}(1)-\mathrm{Fe}-\mathrm{N}(4) & 102.63(8) \\ \mathrm{N}(2)-\mathrm{Fe}-\mathrm{N}(4) & 153.47(8) \\ \mathrm{N}(5)-\mathrm{Fe}-\mathrm{N}(4) & 87.36(8) \\ \mathrm{N}(1)-\mathrm{Fe}-\mathrm{N}(3) & 103.28(8) \\ \mathrm{N}(2)-\mathrm{Fe}-\mathrm{N}(3) & 87.65(8) \\ \mathrm{N}(5)-\mathrm{Fe}-\mathrm{N}(3) & 149.63(8) \\ \mathrm{N}(4)-\mathrm{Fe}-\mathrm{N}(3) & 92.85(8) \\ \mathrm{O}-\mathrm{N}(1)-\mathrm{Fe} & 177.1(2) \\ \mathrm{C}(1)-\mathrm{N}(2)-\mathrm{C}(4) & 108.9(2) \\ \mathrm{C}(1)-\mathrm{N}(2)-\mathrm{Fe} & 117.8(2) \\ \mathrm{C}(4)-\mathrm{N}(2)-\mathrm{Fe} & 133.0(2) \\ \mathrm{C}(6)-\mathrm{N}(3)-\mathrm{C}(9) & 107.1(2) \\ \mathrm{C}(6)-\mathrm{N}(3)-\mathrm{Fe} & 126.2(2) \\ \mathrm{C}(9)-\mathrm{N}(3)-\mathrm{Fe} & 123.0(2) \\ \mathrm{C}(14)-\mathrm{N}(4)-\mathrm{C}(11) & 106.7(2) \\ \mathrm{C}(14)-\mathrm{N}(4)-\mathrm{Fe} & 127.6(2) \\ \mathrm{C}(11)-\mathrm{N}(4)-\mathrm{Fe} & 124.2(1) \\ \mathrm{C}(19)-\mathrm{N}(5)-\mathrm{C}(16) & 108.8(2) \\ \mathrm{C}(19)-\mathrm{N}(5)-\mathrm{Fe} & 117.5(1) \\ \mathrm{C}(16)-\mathrm{N}(5)-\mathrm{Fe} & 132.7(2) \\ \text { a } & \end{array}$

[a]Symmetry transformations used to generate equivalent atoms:

\#1 -x+1,-y,-z+2 \#2 -x+1,-y+1,-z+2 
Table S1e. Anisotropic Displacement Parameters for Fe(TTC)(NO) (TTC = the 5,10,15-tris(4tolyl)corrolato trianion). ${ }^{a}$

\begin{tabular}{|c|c|c|c|c|c|c|}
\hline & $\begin{array}{l}\text { U11 } \\
\left(\AA^{2} \times 10^{3}\right)\end{array}$ & $\mathbf{U} 22\left(\AA^{2} \times 10^{3}\right)$ & $\mathbf{U} 33\left(\AA^{2} \times 10^{3}\right)$ & $\mathbf{U} 23\left(\AA^{2} \times 10^{3}\right)$ & $\mathbf{U} 13\left(\AA^{2} \times 10^{3}\right)$ & $\mathbf{U 1 2}\left(\AA^{2} \times 10^{3}\right)$ \\
\hline $\mathrm{C}(1)$ & $19(1)$ & $24(1)$ & $17(1)$ & $-3(1)$ & $-2(1)$ & $-4(1)$ \\
\hline$C(2)$ & $24(1)$ & $23(1)$ & $20(1)$ & $-2(1)$ & $-2(1)$ & $-5(1)$ \\
\hline$C(3)$ & $19(1)$ & $23(1)$ & $23(1)$ & $-2(1)$ & $0(1)$ & $-2(1)$ \\
\hline $\mathrm{C}(4)$ & $17(1)$ & $21(1)$ & $21(1)$ & $0(1)$ & $-1(1)$ & $-2(1)$ \\
\hline$C(5)$ & $17(1)$ & $21(1)$ & $21(1)$ & $3(1)$ & $-2(1)$ & $-3(1)$ \\
\hline$C(6)$ & $17(1)$ & $20(1)$ & $20(1)$ & $1(1)$ & $-3(1)$ & $-1(1)$ \\
\hline$C(7)$ & $19(1)$ & $25(1)$ & $21(1)$ & $0(1)$ & $-4(1)$ & $0(1)$ \\
\hline $\mathrm{C}(8)$ & $22(1)$ & $29(1)$ & $21(1)$ & $2(1)$ & $-8(1)$ & $-4(1)$ \\
\hline $\mathrm{C}(9)$ & $20(1)$ & $22(1)$ & $19(1)$ & $1(1)$ & $-5(1)$ & $-4(1)$ \\
\hline$C(10)$ & $22(1)$ & $20(1)$ & $18(1)$ & $2(1)$ & $-3(1)$ & $-4(1)$ \\
\hline $\mathrm{C}(11)$ & $22(1)$ & $22(1)$ & $19(1)$ & $1(1)$ & $-5(1)$ & $-4(1)$ \\
\hline$C(12)$ & $24(1)$ & $21(1)$ & $21(1)$ & $-3(1)$ & $-5(1)$ & $0(1)$ \\
\hline$C(13)$ & $22(1)$ & $23(1)$ & $22(1)$ & $1(1)$ & $-4(1)$ & $1(1)$ \\
\hline$C(14)$ & $18(1)$ & $21(1)$ & $21(1)$ & $2(1)$ & $-3(1)$ & $0(1)$ \\
\hline$C(15)$ & $16(1)$ & $25(1)$ & $20(1)$ & $5(1)$ & $-3(1)$ & $-3(1)$ \\
\hline$C(16)$ & $16(1)$ & $22(1)$ & $17(1)$ & $2(1)$ & $-3(1)$ & $-2(1)$ \\
\hline $\mathrm{C}(17)$ & $18(1)$ & $26(1)$ & $19(1)$ & $1(1)$ & $-5(1)$ & $-4(1)$ \\
\hline C(18) & $23(1)$ & $26(1)$ & $19(1)$ & $-1(1)$ & $-6(1)$ & $-6(1)$ \\
\hline C(19) & $23(1)$ & $21(1)$ & $17(1)$ & $-2(1)$ & $-4(1)$ & $-4(1)$ \\
\hline$C(20)$ & $18(1)$ & $25(1)$ & $15(1)$ & $1(1)$ & $-2(1)$ & $0(1)$ \\
\hline $\mathrm{C}(21)$ & $21(1)$ & $25(1)$ & $20(1)$ & $0(1)$ & $-5(1)$ & $-4(1)$ \\
\hline$C(22)$ & $27(1)$ & $23(1)$ & $23(1)$ & $-1(1)$ & $-6(1)$ & $4(1)$ \\
\hline $\mathrm{C}(23)$ & $20(1)$ & $33(1)$ & $19(1)$ & $2(1)$ & $-5(1)$ & $3(1)$ \\
\hline $\mathrm{C}(24)$ & $19(1)$ & $33(1)$ & $24(1)$ & $5(1)$ & $-6(1)$ & $-6(1)$ \\
\hline $\mathrm{C}(25)$ & $23(1)$ & $24(1)$ & $21(1)$ & $2(1)$ & $-4(1)$ & $-2(1)$ \\
\hline$C(26)$ & $24(1)$ & $40(2)$ & $38(2)$ & $0(1)$ & $-6(1)$ & $7(1)$ \\
\hline $\mathrm{C}(27)$ & $19(1)$ & $22(1)$ & $18(1)$ & $-1(1)$ & $-5(1)$ & $2(1)$ \\
\hline $\mathrm{C}(28)$ & $20(1)$ & $25(1)$ & $21(1)$ & $-1(1)$ & $-2(1)$ & $0(1)$ \\
\hline C(29) & $22(1)$ & $25(1)$ & $26(1)$ & $-5(1)$ & $-7(1)$ & $-1(1)$ \\
\hline$C(30)$ & $28(1)$ & $24(1)$ & $20(1)$ & $-1(1)$ & $-8(1)$ & $3(1)$ \\
\hline$C(31)$ & $25(1)$ & $27(1)$ & $17(1)$ & $0(1)$ & $0(1)$ & $-1(1)$ \\
\hline$C(32)$ & $25(1)$ & $28(1)$ & $23(1)$ & $2(1)$ & $-6(1)$ & $-5(1)$ \\
\hline$C(33)$ & $34(2)$ & $38(1)$ & $23(1)$ & $-6(1)$ & $-7(1)$ & $0(1)$ \\
\hline $\mathrm{C}(34)$ & $20(1)$ & $21(1)$ & $22(1)$ & $-3(1)$ & $-5(1)$ & $-1(1)$ \\
\hline$C(35)$ & $25(1)$ & $27(1)$ & $21(1)$ & $1(1)$ & $-3(1)$ & $-1(1)$ \\
\hline$C(36)$ & $19(1)$ & $29(1)$ & $25(1)$ & $-5(1)$ & $2(1)$ & $-1(1)$ \\
\hline$C(37)$ & $21(1)$ & $24(1)$ & $27(1)$ & $-8(1)$ & $-7(1)$ & $0(1)$ \\
\hline $\mathrm{C}(38)$ & $26(1)$ & $30(1)$ & $21(1)$ & $-1(1)$ & $-8(1)$ & $3(1)$ \\
\hline C(39) & $23(1)$ & $30(1)$ & $20(1)$ & $0(1)$ & $-3(1)$ & 1(1) \\
\hline $\mathrm{C}(40)$ & $24(1)$ & $33(1)$ & $34(1)$ & $-8(1)$ & $-10(1)$ & $3(1)$ \\
\hline
\end{tabular}




$\begin{array}{lllllll}\mathrm{C}(41) & 28(2) & 44(2) & 121(4) & 11(2) & 6(2) & 4(2) \\ \mathrm{C}(42) & 78(3) & 58(2) & 37(2) & 2(2) & 5(2) & 23(2) \\ \mathrm{C}(43) & 65(3) & 46(2) & 98(3) & -19(2) & -52(2) & 14(2) \\ \mathrm{C}(44) & 29(2) & 50(2) & 37(2) & 1(1) & 0(1) & -1(1) \\ \mathrm{C}(45) & 32(2) & 49(2) & 31(1) & 9(1) & 4(1) & -3(1) \\ \mathrm{C}(46) & 31(2) & 43(2) & 43(2) & 7(1) & 1(1) & -6(1) \\ \mathrm{Fe} & 16(1) & 21(1) & 16(1) & 0(1) & -4(1) & -1(1) \\ \mathrm{N}(1) & 18(1) & 24(1) & 21(1) & 0(1) & -4(1) & 3(1) \\ \mathrm{N}(2) & 17(1) & 24(1) & 20(1) & 1(1) & -5(1) & -2(1) \\ \mathrm{N}(3) & 20(1) & 21(1) & 16(1) & -2(1) & -2(1) & 0(1) \\ \mathrm{N}(4) & 18(1) & 23(1) & 19(1) & 2(1) & -5(1) & -1(1) \\ \mathrm{N}(5) & 19(1) & 23(1) & 17(1) & 0(1) & -4(1) & -2(1) \\ \mathrm{O} & 34(1) & 39(1) & 47(1) & 21(1) & 3(1) & -2(1)\end{array}$

[a] The anisotropic displacement factor exponent takes the form: $-2 \pi^{2}\left[h^{2} a^{* 2} U_{11}+\ldots+2 h k a * b * U_{12}\right]$ 
Table S1f. Hydrogen Coordinates and Isotropic Displacement Parameters for Fe(TTC)(NO) $(\mathrm{TTC}=$ the 5,10,15-tris(4-tolyl)corrolato trianion).

\begin{tabular}{|c|c|c|c|c|}
\hline & $x\left(x 1^{4}\right)$ & $y\left(x 0^{4}\right)$ & $z\left(x 1^{4}\right)$ & $U_{\text {eq }}\left(\AA^{2} \times 10^{3}\right)$ \\
\hline $\mathrm{H}(2)$ & $10280(30)$ & $2570(30)$ & $4559(19)$ & $37(8)$ \\
\hline $\mathrm{H}(3)$ & $12200(20)$ & $2190(20)$ & $5425(16)$ & $25(7)$ \\
\hline $\mathrm{H}(7)$ & $13320(20)$ & $4410(20)$ & $8422(16)$ & $23(6)$ \\
\hline $\mathrm{H}(8)$ & $12050(30)$ & $6000(20)$ & $9354(18)$ & $33(7)$ \\
\hline $\mathrm{H}(12)$ & $7560(20)$ & $8690(20)$ & 8972(16) & $22(6)$ \\
\hline $\mathrm{H}(13)$ & $5860(20)$ & $8590(20)$ & $7895(15)$ & $18(6)$ \\
\hline $\mathrm{H}(17)$ & $5700(20)$ & $5750(20)$ & $5018(16)$ & $24(6)$ \\
\hline $\mathrm{H}(18)$ & $7340(20)$ & $4100(20)$ & $4424(17)$ & $26(7)$ \\
\hline $\mathrm{H}(21)$ & $12850(20)$ & $1270(20)$ & $6908(15)$ & $20(6)$ \\
\hline $\mathrm{H}(22)$ & $14860(20)$ & $280(20)$ & $6828(15)$ & $21(6)$ \\
\hline $\mathrm{H}(24)$ & $16370(20)$ & $3520(20)$ & $6822(15)$ & $20(6)$ \\
\hline $\mathrm{H}(25)$ & $14400(20)$ & $4560(20)$ & $6896(16)$ & $24(6)$ \\
\hline $\mathrm{H}(28)$ & $11320(20)$ & $8290(20)$ & $9235(16)$ & $17(6)$ \\
\hline $\mathrm{H}(29)$ & $11350(20)$ & $9350(20)$ & $10498(16)$ & $26(7)$ \\
\hline $\mathrm{H}(31)$ & $8190(30)$ & $7870(20)$ & $11427(17)$ & $28(7)$ \\
\hline $\mathrm{H}(32)$ & $8160(20)$ & $6880(20)$ & $10197(15)$ & $20(6)$ \\
\hline $\mathrm{H}(35)$ & $4230(20)$ & $6860(20)$ & $7672(17)$ & $28(7)$ \\
\hline $\mathrm{H}(36)$ & $2240(20)$ & $7820(20)$ & $7376(17)$ & $23(6)$ \\
\hline $\mathrm{H}(38)$ & $3570(30)$ & $9100(30)$ & 5014(19) & $40(8)$ \\
\hline $\mathrm{H}(39)$ & $5530(20)$ & $8120(20)$ & $5235(17)$ & $24(6)$ \\
\hline $\mathrm{H}(41)$ & $6450(40)$ & $1400(40)$ & $9700(30)$ & $71(11)$ \\
\hline $\mathrm{H}(42)$ & $4920(40)$ & $1250(40)$ & $8770(30)$ & $82(13)$ \\
\hline $\mathrm{H}(43)$ & $3540(40)$ & $-170(40)$ & $9020(30)$ & $77(12)$ \\
\hline $\mathrm{H}(44)$ & $5770(30)$ & $3400(30)$ & $9080(20)$ & $57(10)$ \\
\hline $\mathrm{H}(45)$ & $6310(30)$ & $5460(30)$ & $8750(20)$ & $46(8)$ \\
\hline $\mathrm{H}(46)$ & $5570(30)$ & $6970(30)$ & $9690(20)$ & $54(9)$ \\
\hline $\mathrm{H}(26 \mathrm{~A})$ & $17230(30)$ & $270(30)$ & $6594(19)$ & $41(8)$ \\
\hline $\mathrm{H}(33 \mathrm{~A})$ & $8990(30)$ & $9840(30)$ & $12080(20)$ & $52(9)$ \\
\hline $\mathrm{H}(40 \mathrm{~A})$ & $1360(30)$ & $10080(30)$ & $6080(20)$ & $50(9)$ \\
\hline $\mathrm{H}(26 \mathrm{~B})$ & $17780(30)$ & $1550(30)$ & $6450(20)$ & $53(10)$ \\
\hline $\mathrm{H}(33 \mathrm{~B})$ & $9950(30)$ & $8830(30)$ & $12460(20)$ & $43(8)$ \\
\hline $\mathrm{H}(40 \mathrm{~B})$ & $770(30)$ & $8860(30)$ & $6410(20)$ & $46(9)$ \\
\hline $\mathrm{H}(26 \mathrm{C})$ & $17450(40)$ & $990(40)$ & $7400(30)$ & $80(12)$ \\
\hline $\mathrm{H}(33 \mathrm{C})$ & $10410(30)$ & $9930(30)$ & 11933(19) & $43(8)$ \\
\hline $\mathrm{H}(40 \mathrm{C})$ & $1170(30)$ & $9090(20)$ & $5416(19)$ & $36(8)$ \\
\hline
\end{tabular}


Table S2a. Crystal Data and Structure Refinement for Fe(TMOPC)(NO) (TMOPC = the 5,10,15-tris(4-methoxyphenyl)corrolato trianion).

\begin{tabular}{ll}
\hline & Fe(TMOPC)NO \\
\cline { 2 - 2 } Empirical formula & $\mathrm{C}_{46} \mathrm{H}_{35} \mathrm{FeN}_{5} \mathrm{O}_{4}$ \\
Formula weight & 777.64 \\
Temperature & $120(1) \mathrm{K}$ \\
Wavelength & $0.71073 \AA$ \\
Crystal system, space group & Triclinic, P-1 \\
Unit cell dimensions & $a=10.547(4) \AA$ \\
& $b=11.047(5) \AA$ \\
& $c=15.916(7) \AA$ \\
& $\alpha=101.196(7) \mathrm{deg}$. \\
& $\beta=92.972(7) \mathrm{deg}$. \\
& $\mathrm{T}=90.164(7) \mathrm{deg}$. \\
Volume & $1816.5(13) \AA \AA^{3}$ \\
Z, Calculated density & $2,1.422 \mathrm{Mg} / \mathrm{m}^{3}$ \\
Absorption coefficient & $0.470 \mathrm{~mm}{ }^{-1}$ \\
F(000) & 808 \\
Crystal size & $0.15 \times 0.10 \times 0.04 \mathrm{~mm}$ \\
Theta range for data collection & 1.31 to $26.55 \mathrm{deg}$. \\
Limiting indices & $-13<=\mathrm{h}<=13,-13<=\mathrm{k}<=13,-19<=\mathrm{l}<=19$ \\
Reflections collected / unique & $13739 / 7189[\mathrm{R}(\mathrm{int})=0.0685]$ \\
Completeness to theta $=28.15$ & $95.2 \%$ \\
Absorption correction & $\mathrm{None}$ \\
Refinement method & $\mathrm{Full-matrix} \mathrm{least}-\mathrm{squares} \mathrm{on} \mathrm{F}^{2}$ \\
Data / restraints / parameters & $7189 / 0 / 646$ \\
Goodness-of-fit on F ${ }^{2}$ & 1.110 \\
Final R indices [I $>2$ sigma(I) $]$ & $\mathrm{R} 1=0.0708, \mathrm{wR} 2=0.1289$ \\
R indices (all data) & $\mathrm{R} 1=0.1484, \mathrm{wR} 2=0.1461$ \\
Largest diff. peak and hole & 0.781 and $-1.008 \AA^{3}$ \\
&
\end{tabular}


Table S2b. Atomic Coordinates and Equivalent Isotropic Displacement Parameters for $\mathrm{Fe}(\mathrm{TMOPC})(\mathrm{NO})(\mathrm{TMOPC}=$ the 5,10,15-tris(4-methoxyphenyl)corrolato trianion).

\begin{tabular}{|c|c|c|c|c|}
\hline & $x\left(x 1^{4}\right)$ & $y\left(x 10^{4}\right)$ & $\mathrm{z}\left(\mathrm{x} \quad 10^{4}\right)$ & $\mathrm{U}_{\mathrm{eq}}\left(\AA^{2} \times 10^{3}\right)^{a}$ \\
\hline $\mathrm{C}(1)$ & $5592(4)$ & $6322(4)$ & $5658(3)$ & $30(1)$ \\
\hline $\mathrm{C}(2)$ & $4865(4)$ & $7182(4)$ & $5272(3)$ & $31(1)$ \\
\hline $\mathrm{C}(3)$ & $3839(5)$ & $7455(5)$ & $5741(3)$ & $33(1)$ \\
\hline $\mathrm{C}(4)$ & $3887(4)$ & $6777(4)$ & $6422(3)$ & $29(1)$ \\
\hline$C(5)$ & $3023(4)$ & $6635(4)$ & $7022(3)$ & $28(1)$ \\
\hline$C(6)$ & $3219(4)$ & $5788(4)$ & $7593(3)$ & $28(1)$ \\
\hline $\mathrm{C}(7)$ & $2418(5)$ & $5613(5)$ & $8244(3)$ & $32(1)$ \\
\hline $\mathrm{C}(8)$ & $2956(4)$ & $4748(5)$ & $8650(3)$ & $33(1)$ \\
\hline $\mathrm{C}(9)$ & $4106(4)$ & $4376(4)$ & $8254(3)$ & $29(1)$ \\
\hline$C(10)$ & $4964(4)$ & $3480(4)$ & $8464(3)$ & $27(1)$ \\
\hline$C(11)$ & $6073(4)$ & $3160(4)$ & $8046(3)$ & $29(1)$ \\
\hline$C(12)$ & $6926(4)$ & $2183(4)$ & $8153(3)$ & $29(1)$ \\
\hline$C(13)$ & $7860(5)$ & $2170(4)$ & $7611(3)$ & $31(1)$ \\
\hline $\mathrm{C}(14)$ & $7627(4)$ & $3129(4)$ & $7144(3)$ & $31(1)$ \\
\hline$C(15)$ & $8360(4)$ & $3386(4)$ & $6475(3)$ & $29(1)$ \\
\hline$C(16)$ & $7992(4)$ & $4224(4)$ & $5975(3)$ & $30(1)$ \\
\hline$C(17)$ & $8494(5)$ & $4543(4)$ & $5236(3)$ & $31(1)$ \\
\hline $\mathrm{C}(18)$ & $7700(4)$ & $5398(5)$ & $4954(3)$ & $33(1)$ \\
\hline$C(19)$ & $6705(4)$ & $5599(4)$ & $5529(3)$ & $33(1)$ \\
\hline$C(20)$ & $1821(4)$ & $7336(4)$ & $7034(3)$ & $27(1)$ \\
\hline$C(21)$ & $646(5)$ & $6750(5)$ & $6895(3)$ & $31(1)$ \\
\hline$C(22)$ & $-465(5)$ & $7396(5)$ & $6824(3)$ & $34(1)$ \\
\hline $\mathrm{C}(23)$ & $-399(4)$ & $8660(4)$ & $6894(3)$ & $28(1)$ \\
\hline$C(24)$ & $761(4)$ & $9280(5)$ & $7040(3)$ & $33(1)$ \\
\hline$C(25)$ & $1859(5)$ & $8614(5)$ & $7099(3)$ & $34(1)$ \\
\hline$C(26)$ & $-2638(5)$ & $8818(6)$ & $6582(5)$ & $52(2)$ \\
\hline$C(27)$ & $4663(4)$ & $2825(4)$ & $9163(3)$ & $27(1)$ \\
\hline$C(28)$ & $3553(5)$ & $2129(4)$ & $9151(3)$ & $31(1)$ \\
\hline$C(29)$ & $3359(5)$ & $1461(5)$ & $9776(3)$ & $35(1)$ \\
\hline$C(30)$ & $4258(4)$ & $1475(4)$ & $10444(3)$ & $29(1)$ \\
\hline $\mathrm{C}(31)$ & $5350(4)$ & $2190(4)$ & $10489(3)$ & $32(1)$ \\
\hline$C(32)$ & $5542(5)$ & $2857(4)$ & $9857(3)$ & $30(1)$ \\
\hline$C(33)$ & $4904(5)$ & $767(6)$ & $11715(3)$ & $40(1)$ \\
\hline$C(34)$ & $9605(4)$ & $2753(4)$ & $6317(3)$ & $31(1)$ \\
\hline$C(35)$ & $10610(5)$ & $2981(5)$ & $6922(3)$ & $36(1)$ \\
\hline$C(36)$ & $11792(5)$ & $2445(4)$ & $6781(3)$ & $33(1)$ \\
\hline$C(37)$ & $11953(4)$ & $1646(4)$ & $6011(3)$ & $31(1)$ \\
\hline $\mathrm{C}(38)$ & $10958(5)$ & $1374(5)$ & $5391(3)$ & $35(1)$ \\
\hline $\mathrm{C}(39)$ & $9798(5)$ & $1936(4)$ & $5544(3)$ & $33(1)$ \\
\hline$C(40)$ & $14115(6)$ & $1227(6)$ & $6452(4)$ & $50(2)$ \\
\hline$C(41)$ & $940(5)$ & $9141(6)$ & $10048(4)$ & $45(1)$ \\
\hline$C(42)$ & $256(6)$ & $9139(5)$ & $9288(4)$ & $46(2)$ \\
\hline
\end{tabular}




$\begin{array}{llllr}\mathrm{C}(43) & -682(5) & 9986(6) & 9236(4) & 47(2) \\ \mathrm{C}(44) & 9777(6) & 3849(6) & 9517(4) & 48(2) \\ \mathrm{C}(45) & 9200(5) & 4848(5) & 9277(4) & 41(1) \\ \mathrm{C}(46) & 9413(5) & 6007(6) & 9759(4) & 41(1) \\ \mathrm{Fe} & 5865(1) & 5191(1) & 7099(1) & 26(1) \\ \mathrm{N}(1) & 6719(4) & 6243(3) & 7837(2) & 25(1) \\ \mathrm{N}(2) & 4996(3) & 6106(3) & 6366(2) & 25(1) \\ \mathrm{N}(3) & 4271(3) & 5021(3) & 7600(2) & 26(1) \\ \mathrm{N}(4) & 6502(3) & 3715(3) & 7396(2) & 24(1) \\ \mathrm{N}(5) & 6877(3) & 4880(3) & 6145(2) & 28(1) \\ \mathrm{O}(1) & 7374(4) & 6836(3) & 8273(2) & 52(1) \\ \mathrm{O}(2) & -1422(3) & 9402(3) & 6821(2) & 38(1) \\ \mathrm{O}(3) & 3983(3) & 750(3) & 11023(2) & 35(1) \\ \mathrm{O}(4) & 13067(3) & 1023(3) & 5806(2) & 43(1) \\ {[a] \mathrm{U}_{\text {eq }} \text { is defined as one third of the trace of the orthogonalized } \mathrm{U}_{\mathrm{ij}} \text { tensor. }}\end{array}$


Table S2c. Bond Lengths for Fe(TMOPC)(NO) $($ TMOPC $=$ the 5,10,15-tris $(4-$ methoxyphenyl)corrolato trianion). ${ }^{a}$

\begin{tabular}{ll}
\hline \hline Bond & Length $\AA$ \\
\hline $\mathrm{C}(1)-\mathrm{N}(2)$ & $1.378(5)$ \\
$\mathrm{C}(1)-\mathrm{C}(19)$ & $1.423(6)$ \\
$\mathrm{C}(1)-\mathrm{C}(2)$ & $1.432(6)$ \\
$\mathrm{C}(2)-\mathrm{C}(3)$ & $1.348(6)$ \\
$\mathrm{C}(2)-\mathrm{H}(2)$ & $0.98(4)$ \\
$\mathrm{C}(3)-\mathrm{C}(4)$ & $1.431(6)$ \\
$\mathrm{C}(3)-\mathrm{H}(3)$ & $1.10(4)$ \\
$\mathrm{C}(4)-\mathrm{C}(5)$ & $1.383(6)$ \\
$\mathrm{C}(4)-\mathrm{N}(2)$ & $1.384(5)$ \\
$\mathrm{C}(5)-\mathrm{C}(6)$ & $1.435(6)$ \\
$\mathrm{C}(5)-\mathrm{C}(20)$ & $1.486(6)$ \\
$\mathrm{C}(6)-\mathrm{N}(3)$ & $1.399(5)$ \\
$\mathrm{C}(6)-\mathrm{C}(7)$ & $1.412(6)$ \\
$\mathrm{C}(7)-\mathrm{C}(8)$ & $1.363(6)$ \\
$\mathrm{C}(7)-\mathrm{H}(7)$ & $0.97(5)$ \\
$\mathrm{C}(8)-\mathrm{C}(9)$ & $1.422(6)$ \\
$\mathrm{C}(8)-\mathrm{H}(8)$ & $1.05(3)$ \\
$\mathrm{C}(9)-\mathrm{N}(3)$ & $1.388(5)$ \\
$\mathrm{C}(9)-\mathrm{C}(10)$ & $1.419(6)$ \\
$\mathrm{C}(10)-\mathrm{C}(11)$ & $1.389(6)$ \\
$\mathrm{C}(10)-\mathrm{C}(27)$ & $1.486(6)$ \\
$\mathrm{C}(11)-\mathrm{N}(4)$ & $1.393(5)$ \\
$\mathrm{C}(11)-\mathrm{C}(12)$ & $1.437(6)$ \\
$\mathrm{C}(12)-\mathrm{C}(13)$ & $1.341(6)$ \\
$\mathrm{C}(12)-\mathrm{H}(12)$ & $0.86(4)$ \\
$\mathrm{C}(13)-\mathrm{C}(14)$ & $1.423(6)$ \\
$\mathrm{C}(13)-\mathrm{H}(13)$ & $0.92(3)$ \\
$\mathrm{C}(14)-\mathrm{N}(4)$ & $1.394(5)$ \\
$\mathrm{C}(14)-\mathrm{C}(15)$ & $1.419(6)$ \\
$\mathrm{C}(15)-\mathrm{C}(16)$ & $1.379(6)$ \\
$\mathrm{C}(15)-\mathrm{C}(34)$ & $1.499(6)$ \\
$\mathrm{C}(16)-\mathrm{N}(5)$ & $1.394(5)$ \\
$\mathrm{C}(16)-\mathrm{C}(17)$ & $1.418(6)$ \\
$\mathrm{C}(17)-\mathrm{C}(18)$ & $1.388(6)$ \\
$\mathrm{C}(17)-\mathrm{H}(17)$ & $0.95(4)$ \\
$\mathrm{C}(18)-\mathrm{C}(19)$ & $1.418(6)$ \\
$\mathrm{C}(18)-\mathrm{H}(18)$ & $1.13(4)$ \\
$\mathrm{C}(19)-\mathrm{N}(5)$ & $1.382(5)$ \\
$\mathrm{C}(20)-\mathrm{C}(21)$ & $1.385(6)$ \\
$\mathrm{C}(20)-\mathrm{C}(25)$ & $1.396(6)$ \\
$\mathrm{C}(21)-\mathrm{C}(22)$ & $1.385(6)$ \\
$\mathrm{C}(21)-\mathrm{H}(21)$ & $0.85(4)$ \\
&
\end{tabular}




$\begin{array}{ll}\mathrm{C}(22)-\mathrm{C}(23) & 1.379(6) \\ \mathrm{C}(22)-\mathrm{H}(22) & 0.84(4) \\ \mathrm{C}(23)-\mathrm{O}(2) & 1.370(5) \\ \mathrm{C}(23)-\mathrm{C}(24) & 1.388(6) \\ \mathrm{C}(24)-\mathrm{C}(25) & 1.383(6) \\ \mathrm{C}(24)-\mathrm{H}(24) & 1.05(4) \\ \mathrm{C}(25)-\mathrm{H}(25) & 1.04(4) \\ \mathrm{C}(26)-\mathrm{O}(2) & 1.434(6) \\ \mathrm{C}(26)-\mathrm{H}(26 \mathrm{~A}) & 1.04(5) \\ \mathrm{C}(26)-\mathrm{H}(26 \mathrm{~B}) & 1.02(5) \\ \mathrm{C}(26)-\mathrm{H}(26 \mathrm{C}) & 0.97(5) \\ \mathrm{C}(27)-\mathrm{C}(28) & 1.396(6) \\ \mathrm{C}(27)-\mathrm{C}(32) & 1.399(6) \\ \mathrm{C}(28)-\mathrm{C}(29) & 1.371(6) \\ \mathrm{C}(28)-\mathrm{H}(28) & 0.86(4) \\ \mathrm{C}(29)-\mathrm{C}(30) & 1.385(6) \\ \mathrm{C}(29)-\mathrm{H}(29) & 0.92(4) \\ \mathrm{C}(30)-\mathrm{O}(3) & 1.375(5) \\ \mathrm{C}(30)-\mathrm{C}(31) & 1.385(6) \\ \mathrm{C}(31)-\mathrm{C}(32) & 1.380(6) \\ \mathrm{C}(31)-\mathrm{H}(31) & 0.92(3) \\ \mathrm{C}(32)-\mathrm{H}(32) & 0.91(5) \\ \mathrm{C}(33)-\mathrm{O}(3) & 1.427(6) \\ \mathrm{C}(33)-\mathrm{H}(33 \mathrm{~A}) & 1.01(4) \\ \mathrm{C}(33)-\mathrm{H}(33 \mathrm{~B}) & 1.15(5) \\ \mathrm{C}(33)-\mathrm{H}(33 \mathrm{C}) & 0.97(4) \\ \mathrm{C}(34)-\mathrm{C}(35) & 1.383(6) \\ \mathrm{C}(34)-\mathrm{C}(39) & 1.401(6) \\ \mathrm{C}(35)-\mathrm{C}(36) & 1.392(6) \\ \mathrm{C}(35)-\mathrm{H}(35) & 1.02(4) \\ \mathrm{C}(36)-\mathrm{C}(37) & 1.383(6) \\ \mathrm{C}(36)-\mathrm{H}(36) & 0.90(4) \\ \mathrm{C}(37)-\mathrm{O}(4) & 1.384(5) \\ \mathrm{C}(37)-\mathrm{C}(38) & 1.393(6) \\ \mathrm{C}(38)-\mathrm{C}(39) & 1.385(6) \\ \mathrm{C}(38)-\mathrm{H}(38) & 1.03(5) \\ \mathrm{C}(39)-\mathrm{H}(39) & 1.02(4) \\ \mathrm{C}(40)-\mathrm{O}(4) & 1.456(6) \\ \mathrm{C}(40)-\mathrm{H}(40 \mathrm{~A}) & 1.03(4) \\ \mathrm{C}(40)-\mathrm{H}(40 \mathrm{~B}) & 1.09(6) \\ \mathrm{C}(40)-\mathrm{H}(40 \mathrm{C}) & 1.16(5) \\ \mathrm{C}(41)-\mathrm{C}(42) & 1.377(7) \\ \mathrm{C}(41)-\mathrm{C}(43) \# 1 & 1.382(7) \\ \mathrm{C}(41)-\mathrm{H}(41) & 1.00(6) \\ \mathrm{C}(42)-\mathrm{C}(43) & 1.373(7) \\ \mathrm{C}(42)-\mathrm{H}(42) & 0.92(5) \\ & \end{array}$




$\begin{array}{ll}\mathrm{C}(43)-\mathrm{C}(41) \# 1 & 1.382(7) \\ \mathrm{C}(43)-\mathrm{H}(43) & 0.96(6) \\ \mathrm{C}(44)-\mathrm{C}(45) & 1.371(7) \\ \mathrm{C}(44)-\mathrm{C}(46) \# 2 & 1.381(7) \\ \mathrm{C}(44)-\mathrm{H}(44) & 0.88(4) \\ \mathrm{C}(45)-\mathrm{C}(46) & 1.370(7) \\ \mathrm{C}(45)-\mathrm{H}(45) & 1.03(4) \\ \mathrm{C}(46)-\mathrm{C}(44) \# 2 & 1.381(7) \\ \mathrm{C}(46)-\mathrm{H}(46) & 0.82(5) \\ \mathrm{Fe}-\mathrm{N}(1) & 1.702(4) \\ \mathrm{Fe}-\mathrm{N}(5) & 1.878(4) \\ \mathrm{Fe}-\mathrm{N}(2) & 1.890(4) \\ \mathrm{Fe}-\mathrm{N}(4) & 1.900(4) \\ \mathrm{Fe}-\mathrm{N}(3) & 1.922(4) \\ \mathrm{N}(1)-\mathrm{O}(1) & 1.076(4)\end{array}$

[a] Symmetry transformations used to generate equivalent atoms:

\#1 -x,-y+2,-z+2 \#2-x+2,-y+1,-z+2 
Table S2d. Bond Angles for Fe(TMOPC)(NO) (TMOPC = the 5,10,15-tris(4methoxyphenyl)corrolato trianion). ${ }^{a}$

\begin{tabular}{ll}
\hline \hline Bond & Length (deg) \\
\hline $\mathrm{N}(2)-\mathrm{C}(1)-\mathrm{C}(19)$ & $110.6(4)$ \\
$\mathrm{N}(2)-\mathrm{C}(1)-\mathrm{C}(2)$ & $109.1(4)$ \\
$\mathrm{C}(19)-\mathrm{C}(1)-\mathrm{C}(2)$ & $140.2(4)$ \\
$\mathrm{C}(3)-\mathrm{C}(2)-\mathrm{C}(1)$ & $106.7(4)$ \\
$\mathrm{C}(3)-\mathrm{C}(2)-\mathrm{H}(2)$ & $128(2)$ \\
$\mathrm{C}(1)-\mathrm{C}(2)-\mathrm{H}(2)$ & $125(2)$ \\
$\mathrm{C}(2)-\mathrm{C}(3)-\mathrm{C}(4)$ & $109.1(5)$ \\
$\mathrm{C}(2)-\mathrm{C}(3)-\mathrm{H}(3)$ & $127(2)$ \\
$\mathrm{C}(4)-\mathrm{C}(3)-\mathrm{H}(3)$ & $124(2)$ \\
$\mathrm{C}(5)-\mathrm{C}(4)-\mathrm{N}(2)$ & $120.2(4)$ \\
$\mathrm{C}(5)-\mathrm{C}(4)-\mathrm{C}(3)$ & $132.0(5)$ \\
$\mathrm{N}(2)-\mathrm{C}(4)-\mathrm{C}(3)$ & $107.6(4)$ \\
$\mathrm{C}(4)-\mathrm{C}(5)-\mathrm{C}(6)$ & $122.2(4)$ \\
$\mathrm{C}(4)-\mathrm{C}(5)-\mathrm{C}(20)$ & $118.3(4)$ \\
$\mathrm{C}(6)-\mathrm{C}(5)-\mathrm{C}(20)$ & $119.4(4)$ \\
$\mathrm{N}(3)-\mathrm{C}(6)-\mathrm{C}(7)$ & $109.3(4)$ \\
$\mathrm{N}(3)-\mathrm{C}(6)-\mathrm{C}(5)$ & $124.5(4)$ \\
$\mathrm{C}(7)-\mathrm{C}(6)-\mathrm{C}(5)$ & $126.2(4)$ \\
$\mathrm{C}(8)-\mathrm{C}(7)-\mathrm{C}(6)$ & $107.8(4)$ \\
$\mathrm{C}(8)-\mathrm{C}(7)-\mathrm{H}(7)$ & $121(3)$ \\
$\mathrm{C}(6)-\mathrm{C}(7)-\mathrm{H}(7)$ & $131(3)$ \\
$\mathrm{C}(7)-\mathrm{C}(8)-\mathrm{C}(9)$ & $107.7(4)$ \\
$\mathrm{C}(7)-\mathrm{C}(8)-\mathrm{H}(8)$ & $128(2)$ \\
$\mathrm{C}(9)-\mathrm{C}(8)-\mathrm{H}(8)$ & $124(2)$ \\
$\mathrm{N}(3)-\mathrm{C}(9)-\mathrm{C}(10)$ & $123.9(4)$ \\
$\mathrm{N}(3)-\mathrm{C}(9)-\mathrm{C}(8)$ & $109.1(4)$ \\
$\mathrm{C}(10)-\mathrm{C}(9)-\mathrm{C}(8)$ & $126.9(4)$ \\
$\mathrm{C}(11)-\mathrm{C}(10)-\mathrm{C}(9)$ & $123.8(4)$ \\
$\mathrm{C}(11)-\mathrm{C}(10)-\mathrm{C}(27)$ & $117.4(4)$ \\
$\mathrm{C}(9)-\mathrm{C}(10)-\mathrm{C}(27)$ & $118.8(4)$ \\
$\mathrm{C}(10)-\mathrm{C}(11)-\mathrm{N}(4)$ & $124.1(4)$ \\
$\mathrm{C}(10)-\mathrm{C}(11)-\mathrm{C}(12)$ & $127.6(4)$ \\
$\mathrm{N}(4)-\mathrm{C}(11)-\mathrm{C}(12)$ & $108.2(4)$ \\
$\mathrm{C}(13)-\mathrm{C}(12)-\mathrm{C}(11)$ & $108.4(5)$ \\
$\mathrm{C}(13)-\mathrm{C}(12)-\mathrm{H}(12)$ & $128(3)$ \\
$\mathrm{C}(11)-\mathrm{C}(12)-\mathrm{H}(12)$ & $124(3)$ \\
$\mathrm{C}(12)-\mathrm{C}(13)-\mathrm{C}(14)$ & $107.8(5)$ \\
$\mathrm{C}(12)-\mathrm{C}(13)-\mathrm{H}(13)$ & $133(2)$ \\
$\mathrm{C}(14)-\mathrm{C}(13)-\mathrm{H}(13)$ & $119(2)$ \\
$\mathrm{N}(4)-\mathrm{C}(14)-\mathrm{C}(15)$ & $125.0(4)$ \\
$\mathrm{N}(4)-\mathrm{C}(14)-\mathrm{C}(13)$ & $109.2(4)$ \\
$\mathrm{C}(15)-\mathrm{C}(14)-\mathrm{C}(13)$ & $125.6(4)$ \\
&
\end{tabular}




$\begin{array}{ll}\mathrm{C}(16)-\mathrm{C}(15)-\mathrm{C}(14) & 122.6(4) \\ \mathrm{C}(16)-\mathrm{C}(15)-\mathrm{C}(34) & 118.2(4) \\ \mathrm{C}(14)-\mathrm{C}(15)-\mathrm{C}(34) & 119.2(4) \\ \mathrm{C}(15)-\mathrm{C}(16)-\mathrm{N}(5) & 119.1(4) \\ \mathrm{C}(15)-\mathrm{C}(16)-\mathrm{C}(17) & 132.4(4) \\ \mathrm{N}(5)-\mathrm{C}(16)-\mathrm{C}(17) & 108.3(4) \\ \mathrm{C}(18)-\mathrm{C}(17)-\mathrm{C}(16) & 108.5(4) \\ \mathrm{C}(18)-\mathrm{C}(17)-\mathrm{H}(17) & 124(2) \\ \mathrm{C}(16)-\mathrm{C}(17)-\mathrm{H}(17) & 128(2) \\ \mathrm{C}(17)-\mathrm{C}(18)-\mathrm{C}(19) & 106.1(4) \\ \mathrm{C}(17)-\mathrm{C}(18)-\mathrm{H}(18) & 134(2) \\ \mathrm{C}(19)-\mathrm{C}(18)-\mathrm{H}(18) & 120(2) \\ \mathrm{N}(5)-\mathrm{C}(19)-\mathrm{C}(18) & 110.1(4) \\ \mathrm{N}(5)-\mathrm{C}(19)-\mathrm{C}(1) & 111.2(4) \\ \mathrm{C}(18)-\mathrm{C}(19)-\mathrm{C}(1) & 138.7(5) \\ \mathrm{C}(21)-\mathrm{C}(20)-\mathrm{C}(25) & 117.8(4) \\ \mathrm{C}(21)-\mathrm{C}(20)-\mathrm{C}(5) & 121.9(4) \\ \mathrm{C}(25)-\mathrm{C}(20)-\mathrm{C}(5) & 120.0(4) \\ \mathrm{C}(22)-\mathrm{C}(21)-\mathrm{C}(20) & 121.8(5) \\ \mathrm{C}(22)-\mathrm{C}(21)-\mathrm{H}(21) & 118(3) \\ \mathrm{C}(20)-\mathrm{C}(21)-\mathrm{H}(21) & 121(3) \\ \mathrm{C}(23)-\mathrm{C}(22)-\mathrm{C}(21) & 119.1(5) \\ \mathrm{C}(23)-\mathrm{C}(22)-\mathrm{H}(22) & 120(3) \\ \mathrm{C}(21)-\mathrm{C}(22)-\mathrm{H}(22) & 121(3) \\ \mathrm{O}(2)-\mathrm{C}(23)-\mathrm{C}(22) & 124.9(4) \\ \mathrm{O}(2)-\mathrm{C}(23)-\mathrm{C}(24) & 114.4(4) \\ \mathrm{C}(22)-\mathrm{C}(23)-\mathrm{C}(24) & 120.7(5) \\ \mathrm{C}(25)-\mathrm{C}(24)-\mathrm{C}(23) & 119.2(5) \\ \mathrm{C}(25)-\mathrm{C}(24)-\mathrm{H}(24) & 120(2) \\ \mathrm{C}(23)-\mathrm{C}(24)-\mathrm{H}(24) & 121(2) \\ \mathrm{C}(24)-\mathrm{C}(25)-\mathrm{C}(20) & 121.4(4) \\ \mathrm{C}(24)-\mathrm{C}(25)-\mathrm{H}(25) & 119(2) \\ \mathrm{C}(20)-\mathrm{C}(25)-\mathrm{H}(25) & 120(2) \\ \mathrm{O}(2)-\mathrm{C}(26)-\mathrm{H}(26 \mathrm{~A}) & 95(3) \\ \mathrm{O}(2)-\mathrm{C}(26)-\mathrm{H}(26 \mathrm{~B}) & 108(3) \\ \mathrm{H}(26 \mathrm{~A})-\mathrm{C}(26)-\mathrm{H}(26 \mathrm{~B}) & 116(4) \\ \mathrm{O}(2)-\mathrm{C}(26)-\mathrm{H}(26 \mathrm{C}) & 104(3) \\ \mathrm{H}(26 \mathrm{~A})-\mathrm{C}(26)-\mathrm{H}(26 \mathrm{C}) & 111(4) \\ \mathrm{H}(26 \mathrm{~B})-\mathrm{C}(26)-\mathrm{H}(26 \mathrm{C}) & 119(4) \\ \mathrm{C}(28)-\mathrm{C}(27)-\mathrm{C}(32) & 117.4(4) \\ \mathrm{C}(28)-\mathrm{C}(27)-\mathrm{C}(10) & 123.1(4) \\ \mathrm{C}(32)-\mathrm{C}(27)-\mathrm{C}(10) & 119.5(4) \\ \mathrm{C}(29)-\mathrm{C}(28)-\mathrm{C}(27) & 121.2(5) \\ \mathrm{C}(29)-\mathrm{C}(28)-\mathrm{H}(28) & 125(3) \\ \mathrm{C}(27)-\mathrm{C}(28)-\mathrm{H}(28) & 114(3) \\ \mathrm{C}(28)-\mathrm{C}(29)-\mathrm{C}(30) & 120.5(5) \\ & \end{array}$




$\begin{array}{ll}\mathrm{C}(28)-\mathrm{C}(29)-\mathrm{H}(29) & 119(2) \\ \mathrm{C}(30)-\mathrm{C}(29)-\mathrm{H}(29) & 120(2) \\ \mathrm{O}(3)-\mathrm{C}(30)-\mathrm{C}(31) & 124.6(4) \\ \mathrm{O}(3)-\mathrm{C}(30)-\mathrm{C}(29) & 115.8(4) \\ \mathrm{C}(31)-\mathrm{C}(30)-\mathrm{C}(29) & 119.6(4) \\ \mathrm{C}(32)-\mathrm{C}(31)-\mathrm{C}(30) & 119.6(5) \\ \mathrm{C}(32)-\mathrm{C}(31)-\mathrm{H}(31) & 115(2) \\ \mathrm{C}(30)-\mathrm{C}(31)-\mathrm{H}(31) & 125(2) \\ \mathrm{C}(31)-\mathrm{C}(32)-\mathrm{C}(27) & 121.6(5) \\ \mathrm{C}(31)-\mathrm{C}(32)-\mathrm{H}(32) & 120(3) \\ \mathrm{C}(27)-\mathrm{C}(32)-\mathrm{H}(32) & 119(3) \\ \mathrm{O}(3)-\mathrm{C}(33)-\mathrm{H}(33 \mathrm{~A}) & 108(2) \\ \mathrm{O}(3)-\mathrm{C}(33)-\mathrm{H}(33 \mathrm{~B}) & 109(2) \\ \mathrm{H}(33 \mathrm{~A})-\mathrm{C}(33)-\mathrm{H}(33 \mathrm{~B}) & 106(3) \\ \mathrm{O}(3)-\mathrm{C}(33)-\mathrm{H}(33 \mathrm{C}) & 107(3) \\ \mathrm{H}(33 \mathrm{~A})-\mathrm{C}(33)-\mathrm{H}(33 \mathrm{C}) & 120(4) \\ \mathrm{H}(33 \mathrm{~B})-\mathrm{C}(33)-\mathrm{H}(33 \mathrm{C}) & 107(4) \\ \mathrm{C}(35)-\mathrm{C}(34)-\mathrm{C}(39) & 117.9(4) \\ \mathrm{C}(35)-\mathrm{C}(34)-\mathrm{C}(15) & 121.0(4) \\ \mathrm{C}(39)-\mathrm{C}(34)-\mathrm{C}(15) & 121.2(4) \\ \mathrm{C}(34)-\mathrm{C}(35)-\mathrm{C}(36) & 122.3(5) \\ \mathrm{C}(34)-\mathrm{C}(35)-\mathrm{H}(35) & 123(2) \\ \mathrm{C}(36)-\mathrm{C}(35)-\mathrm{H}(35) & 114(2) \\ \mathrm{C}(37)-\mathrm{C}(36)-\mathrm{C}(35) & 118.4(5) \\ \mathrm{C}(37)-\mathrm{C}(36)-\mathrm{H}(36) & 121(3) \\ \mathrm{C}(35)-\mathrm{C}(36)-\mathrm{H}(36) & 121(3) \\ \mathrm{C}(36)-\mathrm{C}(37)-\mathrm{O}(4) & 123.9(4) \\ \mathrm{C}(36)-\mathrm{C}(37)-\mathrm{C}(38) & 121.1(5) \\ \mathrm{O}(4)-\mathrm{C}(37)-\mathrm{C}(38) & 115.0(4) \\ \mathrm{C}(39)-\mathrm{C}(38)-\mathrm{C}(37) & 119.2(5) \\ \mathrm{C}(39)-\mathrm{C}(38)-\mathrm{H}(38) & 125(3) \\ \mathrm{C}(37)-\mathrm{C}(38)-\mathrm{H}(38) & 116(3) \\ \mathrm{C}(38)-\mathrm{C}(39)-\mathrm{C}(34) & 121.1(5) \\ \mathrm{C}(38)-\mathrm{C}(39)-\mathrm{H}(39) & 120(2) \\ \mathrm{C}(34)-\mathrm{C}(39)-\mathrm{H}(39) & 118(2) \\ \mathrm{O}(4)-\mathrm{C}(40)-\mathrm{H}(40 \mathrm{~A}) & 109(2) \\ \mathrm{O}(4)-\mathrm{C}(40)-\mathrm{H}(40 \mathrm{~B}) & 102(3) \\ \mathrm{H}(40 \mathrm{~A})-\mathrm{C}(40)-\mathrm{H}(40 \mathrm{~B}) & 110(4) \\ \mathrm{O}(4)-\mathrm{C}(40)-\mathrm{H}(40 \mathrm{C}) & 103(2) \\ \mathrm{H}(40 \mathrm{~A})-\mathrm{C}(40)-\mathrm{H}(40 \mathrm{C}) & 117(3) \\ \mathrm{H}(40 \mathrm{~B})-\mathrm{C}(40)-\mathrm{H}(40 \mathrm{C}) & 114(4) \\ \mathrm{C}(42)-\mathrm{C}(41)-\mathrm{C}(43) \# 1 & 119.2(6) \\ \mathrm{C}(42)-\mathrm{C}(41)-\mathrm{H}(41) & 119(4) \\ \mathrm{C}(43) \#-\mathrm{C}(4)-\mathrm{C}(42)-\mathrm{C}(41) & 120.8(6) \\ \mathrm{C}(43)-\mathrm{C}(42)-\mathrm{H}(42) & 124(3)\end{array}$




\begin{tabular}{|c|c|}
\hline $\mathrm{C}(41)-\mathrm{C}(42)-\mathrm{H}(42)$ & $115(3)$ \\
\hline$C(42)-C(43)-C(41) \# 1$ & $120.0(6)$ \\
\hline $\mathrm{C}(42)-\mathrm{C}(43)-\mathrm{H}(43)$ & $125(4)$ \\
\hline $\mathrm{C}(41) \# 1-\mathrm{C}(43)-\mathrm{H}(43)$ & $115(4)$ \\
\hline$C(45)-C(44)-C(46) \# 2$ & $120.8(6)$ \\
\hline $\mathrm{C}(45)-\mathrm{C}(44)-\mathrm{H}(44)$ & $120(3)$ \\
\hline $\mathrm{C}(46) \# 2-\mathrm{C}(44)-\mathrm{H}(44)$ & $120(3)$ \\
\hline$C(46)-C(45)-C(44)$ & $120.1(5)$ \\
\hline $\mathrm{C}(46)-\mathrm{C}(45)-\mathrm{H}(45)$ & $125(2)$ \\
\hline C(44)-C(45)-H(45) & $114(2)$ \\
\hline$C(45)-C(46)-C(44) \# 2$ & $119.1(6)$ \\
\hline $\mathrm{C}(45)-\mathrm{C}(46)-\mathrm{H}(46)$ & $125(4)$ \\
\hline $\mathrm{C}(44) \# 2-\mathrm{C}(46)-\mathrm{H}(46)$ & $116(4)$ \\
\hline $\mathrm{N}(1)-\mathrm{Fe}-\mathrm{N}(5)$ & $104.5(2)$ \\
\hline $\mathrm{N}(1)-\mathrm{Fe}-\mathrm{N}(2$ & $105.9(2)$ \\
\hline $\mathrm{N}(5)-\mathrm{Fe}-\mathrm{N}(2$ & $79.4(2)$ \\
\hline $\mathrm{N}(1)-\mathrm{Fe}-\mathrm{N}($ & $99.4(2)$ \\
\hline $\mathrm{N}(5)-\mathrm{Fe}-\mathrm{N}(4)$ & $87.7(2)$ \\
\hline $\mathrm{N}(2)-\mathrm{Fe}-\mathrm{N}(4)$ & $153.8(1)$ \\
\hline $\mathrm{N}(1)-\mathrm{Fe}-\mathrm{N}(3)$ & $105.3(2)$ \\
\hline $\mathrm{N}(5)-\mathrm{Fe}-\mathrm{N}(3)$ & $149.8(1)$ \\
\hline $\mathrm{N}(2)-\mathrm{Fe}-\mathrm{N}(3)$ & $87.5(2)$ \\
\hline $\mathrm{N}(4)-\mathrm{Fe}-\mathrm{N}(3)$ & $92.6(2)$ \\
\hline $\mathrm{O}(1)-\mathrm{N}(1)-\mathrm{Fe}$ & $172.0(4)$ \\
\hline $\mathrm{C}(1)-\mathrm{N}(2)-\mathrm{C}(4)$ & $107.5(4)$ \\
\hline $\mathrm{C}(1)-\mathrm{N}(2)-\mathrm{Fe}$ & $118.4(3)$ \\
\hline $\mathrm{C}(4)-\mathrm{N}(2)-\mathrm{Fe}$ & $133.7(3)$ \\
\hline $\mathrm{C}(9)-\mathrm{N}(3)-\mathrm{C}(6)$ & $106.1(4)$ \\
\hline $\mathrm{C}(9)-\mathrm{N}(3)-\mathrm{Fe}$ & $124.2(3)$ \\
\hline $\mathrm{C}(6)-\mathrm{N}(3)-\mathrm{Fe}$ & \\
\hline $\mathrm{C}(11)-\mathrm{N}(4)-\mathrm{C}(14)$ & $106.3(4)$ \\
\hline $\mathrm{C}(11)-\mathrm{N}(4)-\mathrm{Fe}$ & $125.7(3)$ \\
\hline $\mathrm{C}(14)-\mathrm{N}(4)-\mathrm{Fe}$ & $126.8(3)$ \\
\hline $\mathrm{C}(19)-\mathrm{N}(5)-\mathrm{C}(16)$ & $107.0(4)$ \\
\hline $\mathrm{C}(19)-\mathrm{N}(5)-\mathrm{Fe}$ & $118.2(3)$ \\
\hline $\mathrm{C}(16)-\mathrm{N}(5)-\mathrm{Fe}$ & $133.3(3)$ \\
\hline $\mathrm{C}(23)-\mathrm{O}(2)-\mathrm{C}(26)$ & $117.8(4)$ \\
\hline $\mathrm{C}(30)-\mathrm{O}(3)-\mathrm{C}(33)$ & $115.9(4)$ \\
\hline $\mathrm{C}(37)-\mathrm{O}(4)-\mathrm{C}(40)$ & $117.1(4)$ \\
\hline
\end{tabular}

[a] Symmetry transformations used to generate equivalent atoms:

\#1 -x,-y+2,-z+2 \#2-x+2,-y+1,-z+2 
Table S2e. Anisotropic Displacement Parameters for Fe(TMOPC)(NO) (TMOPC = the 5,10,15tris(4-methoxyphenyl)corrolato trianion). ${ }^{a}$

\begin{tabular}{|c|c|c|c|c|c|c|}
\hline & $\left.\mathrm{U} 11 \AA^{2} \times 10^{3}\right)$ & $\mathbf{U} 22\left(\AA^{2} \times 10^{3}\right)$ & $\mathbf{U} 33\left(\AA^{2} \times 10^{3}\right)$ & $\mathbf{U} 23\left(\AA^{2} \times 10^{3}\right)$ & $\mathrm{U} 13\left(\AA^{2} \times 10^{3}\right)$ & $\mathbf{U 1 2}\left(\AA^{2} \times 10^{3}\right)$ \\
\hline $\mathrm{C}(1)$ & $35(3)$ & $34(3)$ & $18(3)$ & $2(2)$ & $1(2)$ & $-9(2)$ \\
\hline $\mathrm{C}(2)$ & $32(3)$ & $34(3)$ & $28(3)$ & $13(2)$ & $-1(2)$ & $-2(2)$ \\
\hline$C(3)$ & $31(3)$ & $38(3)$ & $31(3)$ & $11(3)$ & $-4(2)$ & $-11(2)$ \\
\hline $\mathrm{C}(4)$ & $26(3)$ & $34(3)$ & $23(3)$ & $0(2)$ & $-1(2)$ & $-9(2)$ \\
\hline$C(5)$ & $26(3)$ & $27(3)$ & $31(3)$ & $8(2)$ & $4(2)$ & $-3(2)$ \\
\hline$C(6)$ & $28(3)$ & $26(3)$ & $29(3)$ & $-1(2)$ & $8(2)$ & $-3(2)$ \\
\hline$C(7)$ & $27(3)$ & $35(3)$ & $31(3)$ & $-3(2)$ & $9(2)$ & $-4(2)$ \\
\hline $\mathrm{C}(8)$ & $35(3)$ & $40(3)$ & $23(3)$ & $6(2)$ & $0(2)$ & $-13(3)$ \\
\hline $\mathrm{C}(9)$ & $22(3)$ & $34(3)$ & $31(3)$ & $3(2)$ & $3(2)$ & $-7(2)$ \\
\hline$C(10)$ & $31(3)$ & $25(3)$ & $24(3)$ & $4(2)$ & $-2(2)$ & $-5(2)$ \\
\hline $\mathrm{C}(11)$ & $28(3)$ & $29(3)$ & $27(3)$ & $1(2)$ & $-2(2)$ & $-7(2)$ \\
\hline$C(12)$ & $30(3)$ & $22(3)$ & $35(3)$ & $8(2)$ & $0(2)$ & $-3(2)$ \\
\hline$C(13)$ & $26(3)$ & $28(3)$ & $38(3)$ & $2(2)$ & $8(2)$ & $1(2)$ \\
\hline$C(14)$ & $32(3)$ & $30(3)$ & $26(3)$ & $-3(2)$ & $1(2)$ & $-12(2)$ \\
\hline$C(15)$ & $32(3)$ & $28(3)$ & $26(3)$ & $2(2)$ & $2(2)$ & $-5(2)$ \\
\hline$C(16)$ & $31(3)$ & $34(3)$ & $22(3)$ & $-5(2)$ & $1(2)$ & $-9(2)$ \\
\hline$C(17)$ & $24(3)$ & $38(3)$ & $29(3)$ & $0(2)$ & $5(2)$ & $-5(2)$ \\
\hline $\mathrm{C}(18)$ & $30(3)$ & $40(3)$ & $29(3)$ & $9(3)$ & $-1(2)$ & $-5(2)$ \\
\hline C(19) & $29(3)$ & $37(3)$ & $30(3)$ & $0(2)$ & $1(2)$ & $-7(2)$ \\
\hline $\mathrm{C}(20)$ & $25(3)$ & $30(3)$ & $27(3)$ & $3(2)$ & $4(2)$ & $0(2)$ \\
\hline $\mathrm{C}(21)$ & $32(3)$ & $25(3)$ & $37(3)$ & $6(3)$ & $1(2)$ & $-4(3)$ \\
\hline$C(22)$ & $23(3)$ & $36(3)$ & $42(3)$ & $6(3)$ & $4(2)$ & $-5(3)$ \\
\hline $\mathrm{C}(23)$ & $25(3)$ & $33(3)$ & $28(3)$ & $7(2)$ & $8(2)$ & $9(2)$ \\
\hline$C(24)$ & $31(3)$ & $30(3)$ & $39(3)$ & $10(2)$ & $3(2)$ & $-8(2)$ \\
\hline$C(25)$ & $26(3)$ & $36(3)$ & $39(3)$ & $7(3)$ & $4(2)$ & $-7(2)$ \\
\hline$C(26)$ & $35(4)$ & $48(4)$ & $73(5)$ & $12(4)$ & $6(3)$ & $3(3)$ \\
\hline $\mathrm{C}(27)$ & $33(3)$ & $28(3)$ & $22(3)$ & $5(2)$ & $6(2)$ & $4(2)$ \\
\hline $\mathrm{C}(28)$ & $33(3)$ & $36(3)$ & $25(3)$ & $7(2)$ & $0(2)$ & $0(2)$ \\
\hline C(29) & $35(3)$ & $38(3)$ & $33(3)$ & $8(3)$ & $4(3)$ & $-9(3)$ \\
\hline$C(30)$ & $36(3)$ & $31(3)$ & $24(3)$ & $13(2)$ & $5(2)$ & $4(2)$ \\
\hline$C(31)$ & $27(3)$ & $42(3)$ & $26(3)$ & $7(3)$ & $-6(2)$ & $-2(2)$ \\
\hline$C(32)$ & $22(3)$ & $34(3)$ & $29(3)$ & $-3(2)$ & $2(2)$ & $-4(2)$ \\
\hline$C(33)$ & $48(4)$ & $39(4)$ & $33(3)$ & $10(3)$ & $-1(3)$ & $2(3)$ \\
\hline$C(34)$ & $29(3)$ & $34(3)$ & $28(3)$ & $-1(2)$ & $0(2)$ & $-6(2)$ \\
\hline$C(35)$ & $34(3)$ & $41(3)$ & $29(3)$ & $0(3)$ & $-1(2)$ & $-7(3)$ \\
\hline$C(36)$ & $30(3)$ & $36(3)$ & $31(3)$ & $6(3)$ & $-2(3)$ & $-7(3)$ \\
\hline$C(37)$ & $35(3)$ & $29(3)$ & $29(3)$ & $8(2)$ & $4(2)$ & $-2(2)$ \\
\hline$C(38)$ & $32(3)$ & $42(3)$ & $31(3)$ & $3(3)$ & $5(3)$ & $4(3)$ \\
\hline C(39) & $32(3)$ & $34(3)$ & $28(3)$ & $-2(2)$ & $-3(2)$ & $-4(2)$ \\
\hline$C(40)$ & $42(4)$ & $67(5)$ & $43(4)$ & $21(3)$ & $-10(3)$ & $-2(3)$ \\
\hline$C(41)$ & $44(3)$ & $51(4)$ & $43(4)$ & $21(3)$ & $-4(3)$ & $-5(3)$ \\
\hline$C(42)$ & $59(4)$ & $37(4)$ & $42(4)$ & $6(3)$ & $3(3)$ & $-9(3)$ \\
\hline
\end{tabular}




\begin{tabular}{lllllll}
$\mathrm{C}(43)$ & $46(4)$ & $54(4)$ & $42(4)$ & $18(3)$ & $-6(3)$ & $-4(3)$ \\
$\mathrm{C}(44)$ & $59(4)$ & $39(4)$ & $44(4)$ & $7(3)$ & $-13(3)$ & $-14(3)$ \\
$\mathrm{C}(45)$ & $41(3)$ & $45(4)$ & $36(3)$ & $4(3)$ & $-6(3)$ & $-7(3)$ \\
$\mathrm{C}(46)$ & $42(4)$ & $43(4)$ & $39(3)$ & $10(3)$ & $-1(3)$ & $0(3)$ \\
$\mathrm{Fe}$ & $25(1)$ & $29(1)$ & $24(1)$ & $3(1)$ & $1(1)$ & $-4(1)$ \\
$\mathrm{N}(1)$ & $30(2)$ & $22(2)$ & $25(2)$ & $7(2)$ & $6(2)$ & $5(2)$ \\
$\mathrm{N}(2)$ & $26(2)$ & $25(2)$ & $23(2)$ & $0(2)$ & $4(2)$ & $1(2)$ \\
$\mathrm{N}(3)$ & $29(2)$ & $28(2)$ & $19(2)$ & $-1(2)$ & $3(2)$ & $1(2)$ \\
$\mathrm{N}(4)$ & $25(2)$ & $29(2)$ & $18(2)$ & $3(2)$ & $4(2)$ & $-7(2)$ \\
$\mathrm{N}(5)$ & $22(2)$ & $29(2)$ & $32(2)$ & $0(2)$ & $-2(2)$ & $-6(2)$ \\
$\mathrm{O}(1)$ & $59(3)$ & $48(3)$ & $46(2)$ & $0(2)$ & $0(2)$ & $2(2)$ \\
$\mathrm{O}(2)$ & $29(2)$ & $31(2)$ & $52(2)$ & $2(2)$ & $5(2)$ & $6(2)$ \\
$\mathrm{O}(3)$ & $38(2)$ & $37(2)$ & $33(2)$ & $13(2)$ & $-3(2)$ & $-4(2)$ \\
$\mathrm{O}(4)$ & $33(2)$ & $58(2)$ & $38(2)$ & $11(2)$ & $2(2)$ & $5(2)$ \\
\hline$a 1 T h$ & $15(2)$
\end{tabular}

[a] The anisotropic displacement factor exponent takes the form: $-2 \pi^{2}\left[\mathrm{~h}^{2} \mathrm{a}^{* 2} \mathrm{U}_{11}+\ldots+2 \mathrm{hka} \mathrm{a}^{*} \mathrm{~b} \mathrm{U}_{12}\right]$ 
Table S2f. Hydrogen Coordinates and Isotropic Displacement Parameters for Fe(TMOPC)(NO) (TMOPC $=$ the 5,10,15-tris(4-methoxyphenyl)corrolato trianion).

\begin{tabular}{|c|c|c|c|c|}
\hline & $x\left(x 1^{4}\right)$ & $y\left(x 1^{4}\right)$ & $z\left(x \quad 10^{4}\right)$ & $U_{e q}\left(\AA^{2} \times 10^{3}\right)$ \\
\hline$\overline{H(2)}$ & $5070(40)$ & $7460(40)$ & $4750(30)$ & $36(13)$ \\
\hline $\mathrm{H}(3)$ & $3090(40)$ & $8110(40)$ & $5640(30)$ & $48(14)$ \\
\hline $\mathrm{H}(7)$ & $1620(50)$ & $6000(50)$ & $8420(30)$ & $69(18)$ \\
\hline $\mathrm{H}(8)$ & $2650(30)$ & $4430(30)$ & $9190(20)$ & 11(10) \\
\hline $\mathrm{H}(12)$ & $6790(30)$ & $1660(30)$ & $8480(20)$ & $17(12)$ \\
\hline $\mathrm{H}(13)$ & $8570(30)$ & $1690(30)$ & $7500(20)$ & $0(9)$ \\
\hline $\mathrm{H}(17)$ & $9260(40)$ & $4280(30)$ & $4980(20)$ & $20(11)$ \\
\hline $\mathrm{H}(18)$ & $7730(40)$ & $5940(30)$ & $4420(30)$ & $34(12)$ \\
\hline $\mathrm{H}(21)$ & $580(30)$ & $5980(30)$ & $6860(20)$ & $15(12)$ \\
\hline $\mathrm{H}(22)$ & $-1180(40)$ & $7030(30)$ & $6750(20)$ & $17(12)$ \\
\hline $\mathrm{H}(24)$ & $820(30)$ & $10230(40)$ & $7050(20)$ & $28(11)$ \\
\hline $\mathrm{H}(25)$ & $2730(40)$ & $9090(40)$ & $7210(30)$ & $41(13)$ \\
\hline $\mathrm{H}(28)$ & $3010(40)$ & $2180(40)$ & $8730(30)$ & $41(15)$ \\
\hline H(29) & $2610(40)$ & $1040(30)$ & $9770(20)$ & $17(11)$ \\
\hline $\mathrm{H}(31)$ & $5970(30)$ & $2290(30)$ & $10930(20)$ & $13(10)$ \\
\hline $\mathrm{H}(32)$ & $6270(40)$ & $3300(40)$ & $9870(30)$ & $51(15)$ \\
\hline $\mathrm{H}(35)$ & $10550(40)$ & $3540(40)$ & $7510(30)$ & $35(13)$ \\
\hline $\mathrm{H}(36)$ & $12450(40)$ & $2660(40)$ & $7160(30)$ & $48(15)$ \\
\hline $\mathrm{H}(38)$ & $11190(40)$ & $860(40)$ & $4810(30)$ & $58(16)$ \\
\hline $\mathrm{H}(39)$ & $9080(40)$ & $1810(30)$ & $5080(20)$ & $27(12)$ \\
\hline $\mathrm{H}(41)$ & $1620(60)$ & $8520(60)$ & $10070(40)$ & $110(20)$ \\
\hline $\mathrm{H}(42)$ & $450(50)$ & $8520(50)$ & $8840(30)$ & $70(20)$ \\
\hline $\mathrm{H}(43)$ & $-1230(60)$ & $9990(50)$ & $8740(40)$ & $100(20)$ \\
\hline $\mathrm{H}(44)$ & $9610(40)$ & $3110(40)$ & $9220(30)$ & $27(13)$ \\
\hline $\mathrm{H}(45)$ & $8740(40)$ & $4660(40)$ & $8680(30)$ & $42(13)$ \\
\hline $\mathrm{H}(46)$ & $9060(50)$ & $6640(50)$ & $9670(40)$ & $80(20)$ \\
\hline $\mathrm{H}(26 \mathrm{~A})$ & $-3130(50)$ & $9630(50)$ & $6590(30)$ & $76(19)$ \\
\hline $\mathrm{H}(33 \mathrm{~A})$ & $4630(40)$ & $130(40)$ & $12050(30)$ & $38(13)$ \\
\hline $\mathrm{H}(40 \mathrm{~A})$ & $13840(30)$ & $930(30)$ & $6990(20)$ & $19(11)$ \\
\hline $\mathrm{H}(26 \mathrm{~B})$ & $-2570(40)$ & $8250(40)$ & $6000(30)$ & $47(15)$ \\
\hline $\mathrm{H}(33 \mathrm{~B})$ & $5860(50)$ & $440(40)$ & $11440(30)$ & $64(16)$ \\
\hline $\mathrm{H}(40 \mathrm{~B})$ & $14840(60)$ & $620(60)$ & $6140(40)$ & $100(20)$ \\
\hline $\mathrm{H}(26 \mathrm{C})$ & $-2830(40)$ & $8450(40)$ & $7070(30)$ & $52(17)$ \\
\hline $\mathrm{H}(33 \mathrm{C})$ & $5020(40)$ & $1620(40)$ & $12000(30)$ & $43(15)$ \\
\hline $\mathrm{H}(40 \mathrm{C})$ & $14350(40)$ & $2270(50)$ & $6520(30)$ & $67(16)$ \\
\hline
\end{tabular}


Table S-3: Computational results for $[\mathrm{Fe}(\mathrm{C})(\mathrm{NO})]$

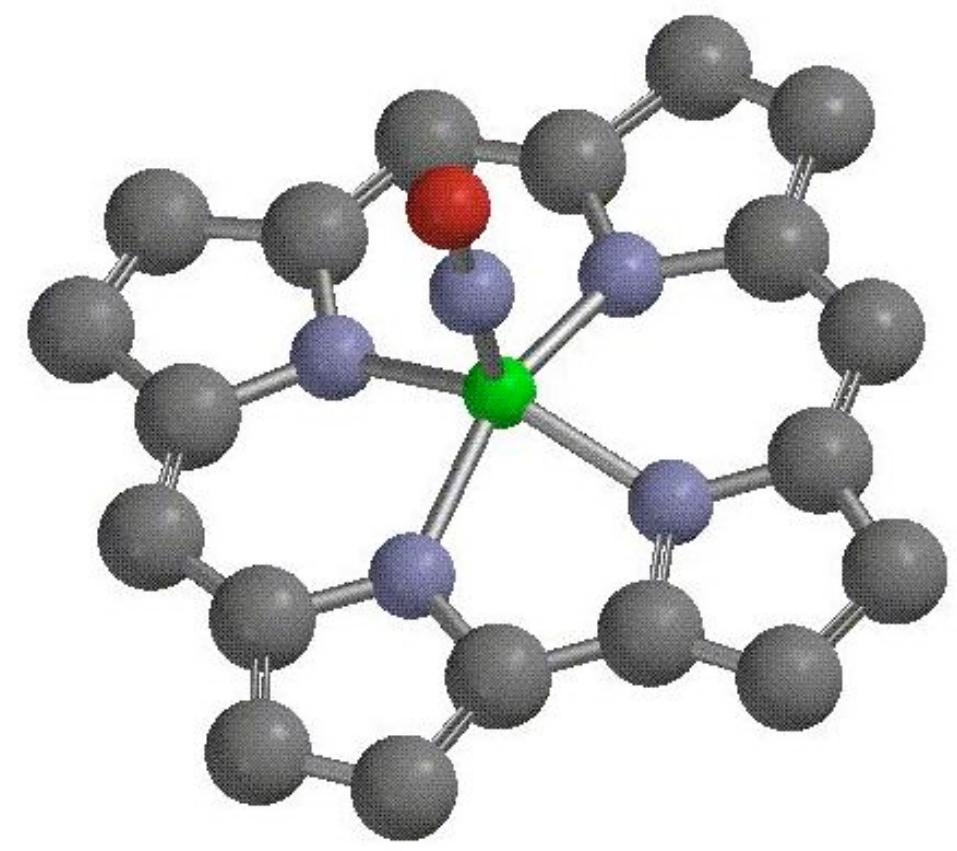

Spartan '04 Quantum Mechanics Program: (PC/x86) Release 121

Job type: Geometry optimization

Method: RB3LYP

Basis set: LACVP*

SCF total energy: -1203.0733004 hartrees.

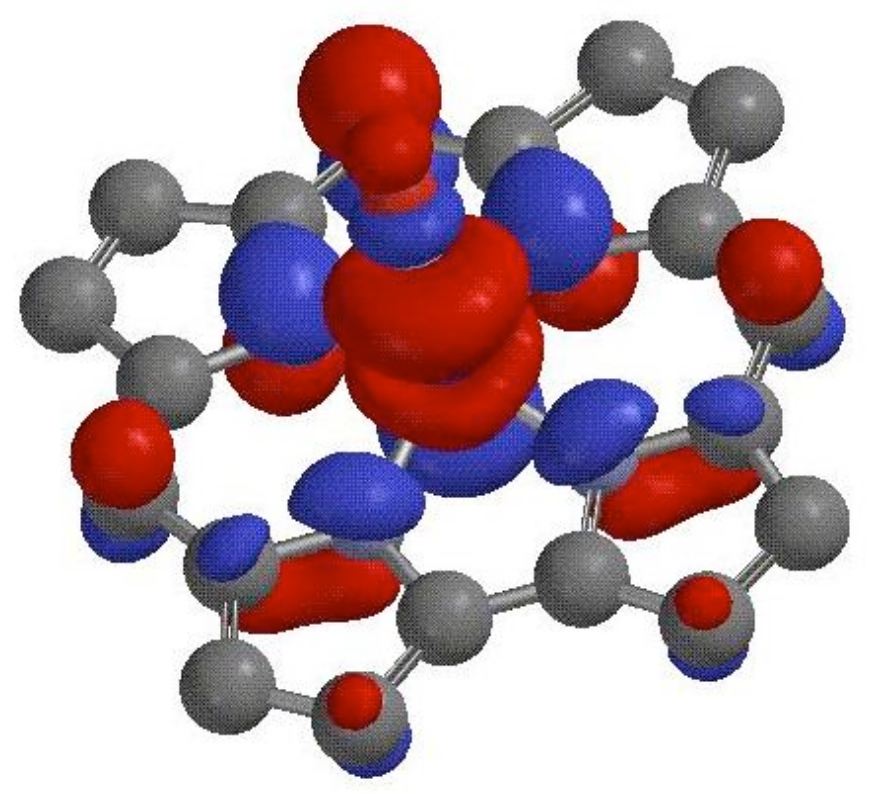

LUMO 


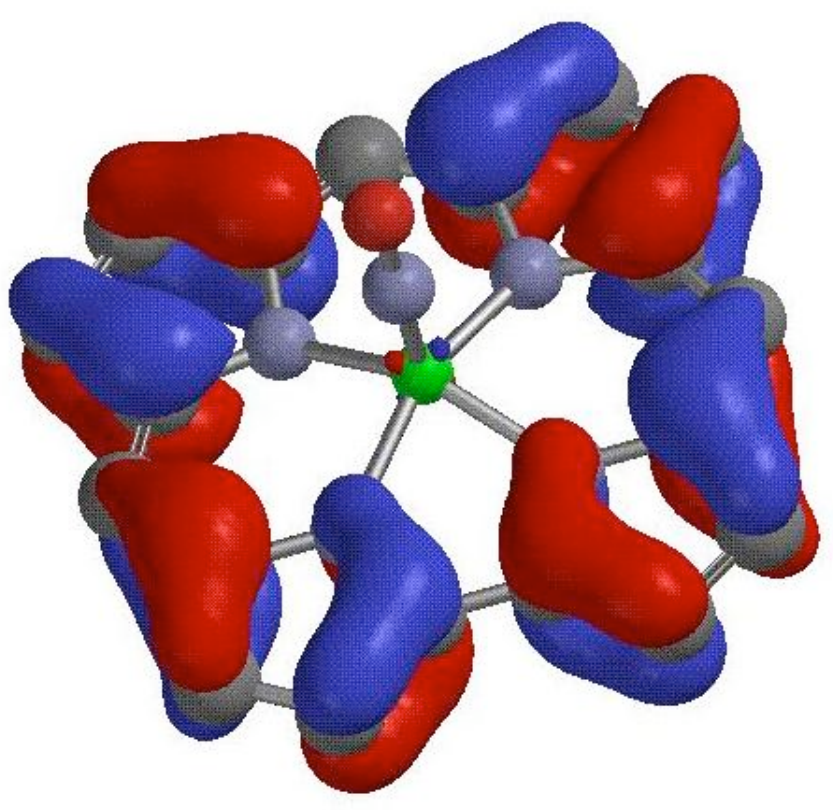

HOMO

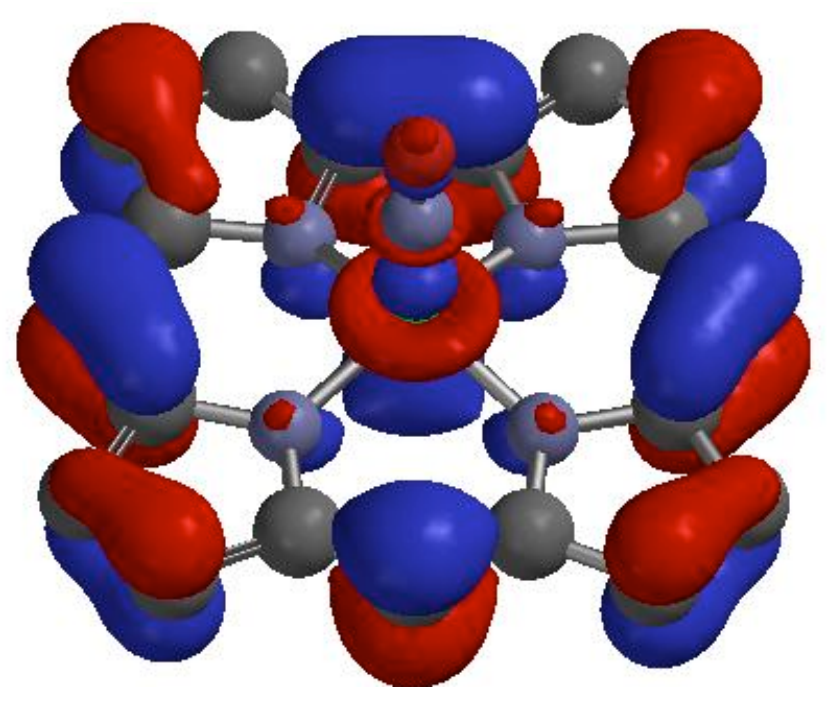

HOMO-1 
Final Geometry:

\begin{tabular}{|c|c|c|c|}
\hline \multicolumn{4}{|c|}{ Cartesian Coordinates (Angstroms) } \\
\hline Atom & $\mathrm{X}$ & $\mathrm{Y} \quad \mathrm{Z}$ & \\
\hline $1 \mathrm{C} \mathrm{C}$ & -0.9982543 & -2.7447831 & 0.0832759 \\
\hline $2 \mathrm{C} \mathrm{C}$ & -2.2532667 & -3.4401259 & 1637116 \\
\hline $3 \mathrm{C} \mathrm{C}$ & -3.2467927 & -2.4995329 & 0.2072692 \\
\hline $4 \mathrm{C} \mathrm{C}$ & -2.6141058 & -1.2130005 & 0.1477974 \\
\hline $5 \mathrm{C} \mathrm{C}$ & 0.2760513 & -3.3339569 & 0.0652318 \\
\hline $6 \mathrm{C} \mathrm{C}$ & -3.2369148 & 0.0324834 & 0.1744407 \\
\hline $7 \mathrm{C} \mathrm{C}$ & 1.4547890 & -2.6031468 & 1362 \\
\hline $8 \mathrm{C} \mathrm{C}$ & 2.8336220 & -2.9909483 & 7934 \\
\hline $9 \mathrm{C} \mathrm{C}$ & 3.5910693 & -1.8360594 & \\
\hline $10 \mathrm{C} \mathrm{C}$ & 2.6811702 & -0.7365686 & 5128 \\
\hline $11 \mathrm{C} \mathrm{C}$ & & & 1459 \\
\hline $12 \mathrm{C} \mathrm{C}$ & $1.50^{\circ}$ & 726544 & 649 \\
\hline $13 \mathrm{C} \mathrm{C}$ & 0.34 & 272490 & 142 \\
\hline $14 \mathrm{C} \mathrm{C}$ & -0.94 & 0400 & 822 \\
\hline $15 \mathrm{C} \mathrm{C}$ & -2.18 & 3390 & 316 \\
\hline $16 \mathrm{C} \mathrm{C}$ & -3.19 & 9458 & 131 \\
\hline $17 \mathrm{C} \mathrm{C}$ & -2.5888186 & 1.2651757 & 1460 \\
\hline $18 \mathrm{H} \mathrm{H}$ & -2.35 & -4.5172636 & 7740 \\
\hline $19 \mathrm{H} \mathrm{H}$ & -4.31 & -2.6608498 & 9588 \\
\hline $20 \mathrm{H} \mathrm{H}$ & & & \\
\hline $21 \mathrm{H} \mathrm{H}$ & -2.2648978 & 4.5631243 & 9611 \\
\hline $22 \mathrm{H} \mathrm{H}$ & 4.6668277 & -1.7637549 & 3790 \\
\hline $23 \mathrm{H} \mathrm{H}$ & 3.1941366 & -4.0100626 & 3913 \\
\hline $24 \mathrm{~N} \mathrm{~N}$ & 1.4359922 & 1.1948996 & 0.0460225 \\
\hline $25 \mathrm{~N} \mathrm{~N}$ & 1.4115390 & -1.2241137 & 1386 \\
\hline $26 \mathrm{~N} \mathrm{~N}$ & -1.2411906 & -1.3832633 & 06139 \\
\hline $27 \mathrm{~N} \mathrm{~N}$ & -1.2127544 & 1.4077821 & 0.0891908 \\
\hline $28 \mathrm{H} \mathrm{H}$ & -4.3217444 & 0.0433985 & 0.2325726 \\
\hline $29 \mathrm{H} \mathrm{H}$ & & & \\
\hline $30 \mathrm{H} \mathrm{H}$ & 0.3280051 & -4.4184167 & 0.0906258 \\
\hline $31 \mathrm{C} \mathrm{C}$ & 3.6277039 & 1.7614279 & 0.2058280 \\
\hline $32 \mathrm{H} \mathrm{H}$ & 4.7018749 & 1.6667468 & 0.2996480 \\
\hline $33 \mathrm{C} \mathrm{C}$ & 2.8939502 & 2.9317044 & 0.1797637 \\
\hline $34 \mathrm{H} \mathrm{H}$ & 3.2751877 & 3.9429424 & 0.2452317 \\
\hline $35 \mathrm{Fe} \mathrm{Fe}$ & 0.0206025 & 0.0009189 & -0.3707865 \\
\hline $36 \mathrm{~N} \mathrm{~N}$ & -0.0403481 & 0.0062214 & -1.9733242 \\
\hline $37 \mathrm{O} O$ & -0.0863877 & 0.0088400 & -3.1331532 \\
\hline
\end{tabular}

